LA-14157

Approved for public release;

distribution is unlimited.

A Weighted Point Model for the

Thermal Neutron Multiplicity Assay of

High-Mass Plutonium Samples

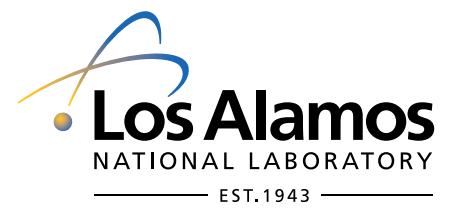

The World's Greatest Science Protecting America 
Los Alamos National Laboratory, an affirmative action/equal opportunity employer, is operated by the University of California for the United States Department of Energy under contract W-7405-ENG-36.

This report was prepared as an account of work sponsored by an agency of the United States Government. Neither the Regents of the University of California, the United States Government nor any agency thereof, nor any of their employees make any warranty, express or implied, or assume any legal liability or responsibility for the accuracy, completeness, or usefulness of any information, apparatus, product, or process disclosed, or represent that its use would not infringe privately owned rights. Reference herein to any specific commercial product, process, or service by trade name, trademark, manufacturer, or otherwise does not necessarily constitute or imply its endorsement, recommendation, or favoring by the Regents of the University of California, the United States Government, or any agency thereof. The views and opinions of authors expressed herein do not necessarily state or reflect those of the Regents of the University of California, the United States Government, or any agency thereof. Los Alamos National Laboratory strongly supports academic freedom and a researcher's right to publish; as an institution, however, the Laboratory does not endorse the viewpoint of a publication or guarantee its technical correctness. 
LA-14157

Issued: October 2005

\title{
A Weighted Point Model for the \\ Thermal Neutron Multiplicity Assay of \\ High-Mass Plutonium Samples
}

\author{
Merlyn S. Krick \\ William H. Geist \\ Douglas R. Mayo
}





\section{TABLE OF CONTENTS}

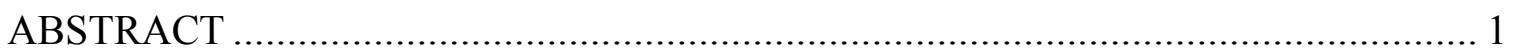

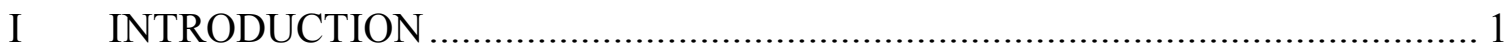

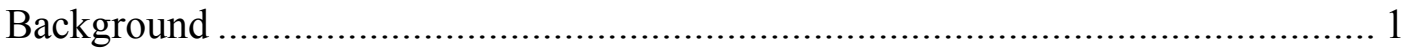

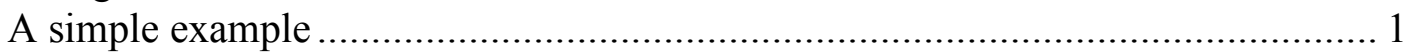

Multiplication variations in plutonium samples............................................. 3

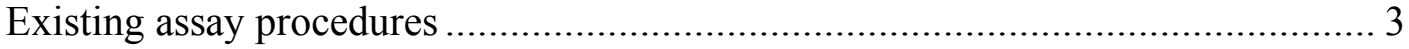

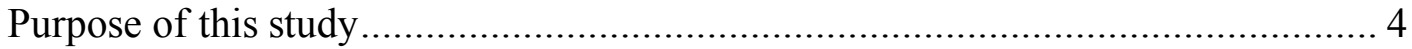

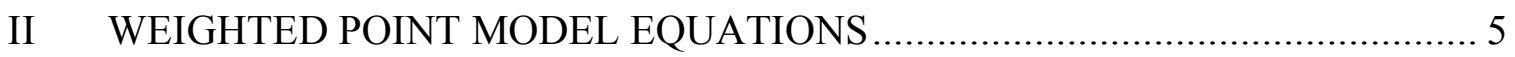

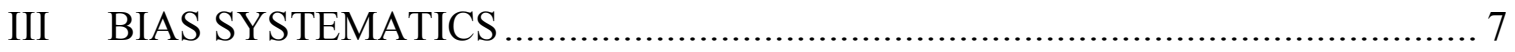

IV WEIGHTED POINT MODEL SIMULATED ASSAYS …………………….... 14

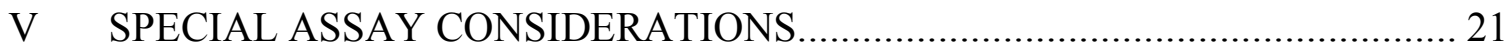

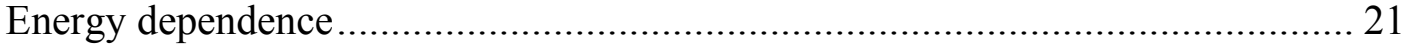

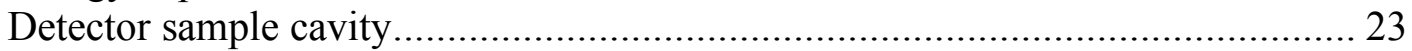

Sensitivity to detector parameters ………………............................................. 24

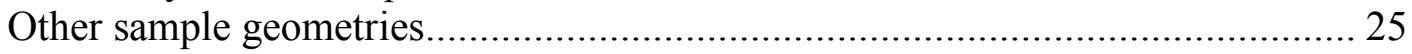

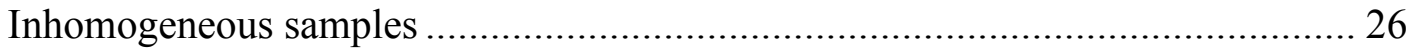

VI COMPARISON WITH EXPERIMENT_....................................................... 27

Comparison with measurement data from a plutonium oxide sample with known mass and isotopic composition ................................................. 27

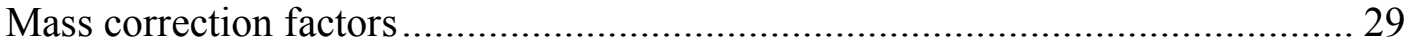

Alpha dependence of the mass correction factors .............................................. 30

Assay of Rocky Flats plutonium metal samples ................................................... 32

Assay of Savannah River plutonium metal samples ............................................ 35

VII FOUR-PARAMETER POINT MODEL MULTIPLICITY ANALYSIS ............... 37

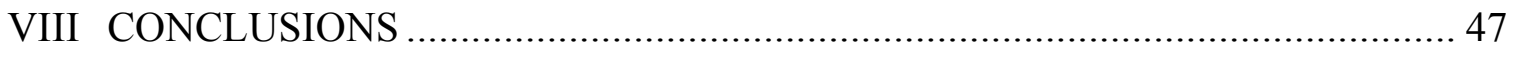

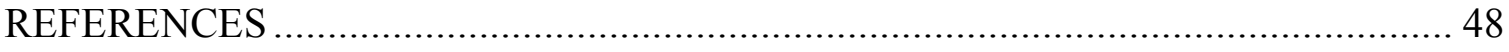




\section{LIST OF FIGURES}

Figure 1. Leakage multiplication vs. position for a 2-kg plutonium metal cylinder with diameter $=$ height.

Figure 2. Triples weighting factor vs. leakage multiplication for a 2-kg plutonium metal cylinder with various height/diameter ratios.

Figure 3. Triples weighting factor vs. leakage multiplication for plutonium metal cylinders with a ${ }^{240} \mathrm{Pu}$ spontaneous fission source and masses from 0.25 to $4 \mathrm{~kg}$............ 11 Figure 4. Triples weighting factor vs. leakage multiplication for plutonium metal cylinders with masses from 0.25 to $4 \mathrm{~kg}$

Figure 5. Doubles weighting factor vs. leakage multiplication for plutonium metal cylinders with a ${ }^{240} \mathrm{Pu}$ spontaneous fission source and masses from 0.25 to $4 \mathrm{~kg}$............ 13 Figure 6. Doubles weighting factor vs. leakage multiplication for plutonium metal cylinders with masses from 0.25 to $4 \mathrm{~kg}$.

Figure 7. Ratio of assay to true effective ${ }^{240} \mathrm{Pu}$ mass vs. sample number for the 66 plutonium cylinders used to determine the doubles and triples weighting factors.

Figure 8. Assay alpha value vs. sample number for the 66 plutonium cylinders used to determine the doubles and triples weighting factors.

Figure 9. Ratio of assay to true multiplication vs. sample number for the 66 plutonium

cylinders used to determine the doubles and triples weighting factors.

Figure 10. Ratio of assay to true multiplication vs. true effective ${ }^{240} \mathrm{Pu}$ mass for 25 randomized plutonium metal samples.

Figure 11. Alpha value difference (assay minus true) vs. true effective ${ }^{240} \mathrm{Pu}$ mass for 25 randomized plutonium metal samples.............................................................. 20 Figure 12. Ratio of assay to true effective ${ }^{240} \mathrm{Pu}$ mass vs. true effective ${ }^{240} \mathrm{Pu}$ mass for 25 randomized plutonium metal samples.

Figure 13. Ratio of assay to true effective ${ }^{240} \mathrm{Pu}$ mass vs. energy for a monoenergetic $(\alpha, n)$ neutron source

Figure 14. Correction factors for the leakage multiplication and effective ${ }^{240} \mathrm{Pu}$ mass vs. detector cavity size for a $2-\mathrm{kg}$ plutonium metal cylinder with diameter $=$ height centered in the cavity.

Figure 15. Ratio of assay to true effective ${ }^{240} \mathrm{Pu}$ mass vs. assay alpha value for a plutonium sample with a multiplication of 2 assayed with estimated detector parameters.

Figure 16. Effective ${ }^{240} \mathrm{Pu}$ mass correction factor vs. leakage multiplication for the 66 plutonium metal cylinders used to determine the doubles and triples weighting factors.

Figure 17. Ratio of assay to true mass vs. $(\alpha, \mathrm{n})$ fill fraction $(f)$ for a $2-\mathrm{kg}$ plutonium metal cylinder with diameter $=$ height.

Figure 18. Effective ${ }^{240} \mathrm{Pu}$ mass correction factor vs. measured multiplication for two sets of plutonium samples - one real and one computational.

Figure 19. Effective ${ }^{240} \mathrm{Pu}$ mass correction factor vs. measured alpha value from

MCNPX and experimental data.

Figure 20. Ratio of assay to reference effective ${ }^{240} \mathrm{Pu}$ mass vs. reference effective

${ }^{240} \mathrm{Pu}$ mass for 32 Rocky Flats samples measured in the FBLNMC and analyzed with the standard point model. 
Figure 21. Ratio of assay to reference effective ${ }^{240} \mathrm{Pu}$ mass vs. reference effective ${ }^{240} \mathrm{Pu}$ mass for 32 Rocky Flats samples measured in the FBLNMC and analyzed with the weighted point model.

Figure 22. Ratio of assay to reference effective ${ }^{240} \mathrm{Pu}$ mass vs. measured alpha for 32 Rocky Flats samples measured in the FBLNMC and analyzed with the weighted point

model. 23 . Ratio of assay to reference effective ${ }^{240} \mathrm{Pu}$ mass vs. reference effective ${ }^{240} \mathrm{Pu}$ mass for six well-characterized Savannah River plutonium metal samples measured in the FBLNMC and analyzed with the standard and weighted point models.

Figure 24. Ratio of assay to reference effective ${ }^{240} \mathrm{Pu}$ mass vs. measured alpha for six well-characterized Savannah River plutonium metal samples measured in the FBLNMC and analyzed with the weighted point model.

Figure 25. Quads weighting factor vs. leakage multiplication for plutonium metal cylinders with a ${ }^{240} \mathrm{Pu}$ spontaneous fission source and masses from 0.25 to $4 \mathrm{~kg}$.....

Figure 26. Quads weighting factor vs. leakage multiplication for plutonium metal cylinders with masses from 0.25 to $4 \mathrm{~kg}$.

Figure 27. Fill fraction weighting factors for $S, D, T$, and $Q$ vs. the $(\alpha, \mathrm{n})$ fill fraction (f) for a $2-\mathrm{kg}$ plutonium metal cylinder with diameter $=$ height.

Figure 28. The ratio $D Q / T^{2}$ vs. leakage multiplication for three sets of plutonium metal samples.

\section{LIST OF TABLES}

Table I. Data for two idealized plutonium samples

Table II. Spontaneous and induced fission factorial moments for the INCC code and for the point model calculations with the MCNPX data. ............................................. 9

Table III. Data for 25 randomized samples........................................................... 18

Table IV. Detector parameters derived from ${ }^{252} \mathrm{Cf}$ and plutonium............................... 24

Table V. The $S, D, T$, and $Q$ rates for a 2-kg PU cylinder with $m=1, M=1.774, \alpha=1$, and $f=0.2$ from the weighted point model equations and directly from MCNPX

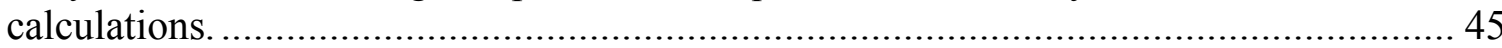

Table VI. Assay results from the weighted point model quad equations using the $S, D, T$, and $Q$ rates from the same equations and from direct MCNPX calculations. 45 Table VII. Assay results obtained by increasing the weighted point model reference rates separately by $0.5 \%$.

Table VIII. Standard deviations of several quantities for simulated 1000-s measurements of the test sample in a sample in a typical thermal neutron multiplicity counter. 


\title{
A WEIGHTED POINT MODEL FOR THE THERMAL NEUTRON MULTIPLICITY ASSAY OF HIGH-MASS PLUTONIUM SAMPLES
}

\author{
M. S. Krick, W. H. Geist, and D. R. Mayo
}

\begin{abstract}
A weighted point model for thermal neutron multiplicity counting has been developed for the assay of impure plutonium metal samples. Weighting factors are introduced for the spontaneous fission and $(\alpha, n)$ contributions to the doubles and triples rates to account for the variations in neutron multiplication in these samples. The weighting factors are obtained from Monte Carlo simulations using the MCNPX code, which supports the simulation of spontaneous fission sources and can tally the source and detected neutron multiplicity distributions. Systematic behavior of the weighting factors was studied as a function of sample mass and geometry. Simulations were performed to evaluate the potential accuracy of assays performed with weighted point model analysis. Comparisons with experimental data are presented. The possible use of quads rates is explored.
\end{abstract}

\section{INTRODUCTION}

\section{Background}

Over the past twenty years, thermal neutron multiplicity counting has become a common nondestructive assay technique for impure plutonium materials [1]. In most applications, the analysis is performed with the three-parameter point model multiplicity equations [2]; the neutron multiplication, alpha value [the ratio of $(\alpha, n)$ to spontaneous fission neutrons], and the effective ${ }^{240} \mathrm{Pu}$ mass [1] are calculated from the measured singles, doubles, and triples count rates corrected for deadtime and background. The point model is based on a number of simplifying assumptions, the most important of which are the assumptions that all neutrons have the same energy and have the same probability for inducing a fission (constant neutron multiplication). Neutrons from $(\alpha, n)$ reactions can have energies much different from fission neutrons, and this can lead to significant bias in the assay masses [3]. In plutonium metal samples and in high-mass, high-density plutonium oxide samples, the neutron multiplication can vary substantially throughout a sample and also lead to significant assay biases.

\section{A simple example}

Consider a thermal neutron multiplicity counter with an efficiency of $50 \%$, doubles gate fraction of 0.7 , and triples gate fraction of 0.5 . Table I shows the effective ${ }^{240} \mathrm{Pu}$ mass $(m)$, neutron multiplication $(M)$, and alpha value $(\alpha)$ for two idealized plutonium samples, which are the same except for the neutron multiplication. 
Table I. Data for two idealized plutonium samples.

\begin{tabular}{|c|c|c|}
\hline & Sample 1 & Sample 2 \\
\hline$m(\mathrm{~g})$ & 100 & 100 \\
\hline$M$ & 1.9 & 2.1 \\
\hline$\alpha$ & 0 & 0 \\
\hline$S\left(\mathrm{~s}^{-1}\right)$ & 96892 & 107091 \\
\hline$D\left(\mathrm{~s}^{-1}\right)$ & 167128 & 234150 \\
\hline$T\left(\mathrm{~s}^{-1}\right)$ & 458976 & 846373 \\
\hline
\end{tabular}

The measured singles rate $(S)$, doubles rate $(D)$, and triples rate $(T)$ for these samples (assuming zero background and deadtime) can be calculated from the point model multiplicity equations using the default spontaneous fission and induced fission factorial moments from the INCC (International Neutron Coincidence Counting) code [4]. These rates are shown in Table I. If both samples are placed in the detector cavity at the same time and sufficiently far apart that they do not interact significantly with each other, then the measured count rates for the two samples will be the sum of the rates shown in Table I:

$$
\begin{aligned}
& S=203983 \\
& D=401278 \\
& T=1305349 .
\end{aligned}
$$

Solving the point model equations with these summed rates for the neutron multiplication, alpha value, and effective ${ }^{240} \mathrm{Pu}$ mass gives

$$
\begin{aligned}
& M=2.019 \\
& \alpha=0.045 \\
& m=189.6 \mathrm{~g} .
\end{aligned}
$$

The true values for the combined samples are

$$
\begin{aligned}
& M=2 \\
& \alpha=0 \\
& m=200 \mathrm{~g},
\end{aligned}
$$

so the point model solution overestimates $M$ and $\alpha$ and underestimates $m$. The assay mass bias is $-5.2 \%$. Although more complex, this general behavior is seen in real plutonium samples because the multiplication varies throughout the sample. Negative bias in the assay masses of $20 \%$ or more is common.

Originally IAEA (International Atomic Energy Agency). 


\section{Multiplication variations in plutonium samples}

To illustrate the multiplication variations in plutonium samples, Monte Carlo calculations were done with the MCNPX code, Version 2.5.B [5] for a $2 \mathrm{~kg}$ plutonium metal cylinder with diameter $=$ height, with density $=18 \mathrm{~g} / \mathrm{cm}^{3}$, and with $94 \%{ }^{239} \mathrm{Pu}$ and $6 \%{ }^{240} \mathrm{Pu}$. A point neutron source was used with a ${ }^{240} \mathrm{Pu}$ spontaneous fission neutron energy spectrum. Leakage neutron multiplication (neutrons escaping the sample per source neutron) was calculated for point neutron sources along a straight line extending from the center of the cylinder to the top outer edge of the cylinder. Fig. 1 shows the leakage multiplication vs. position. The leakage multiplication decreases from 2.22 at the center to 1.26 at the top outer edge.

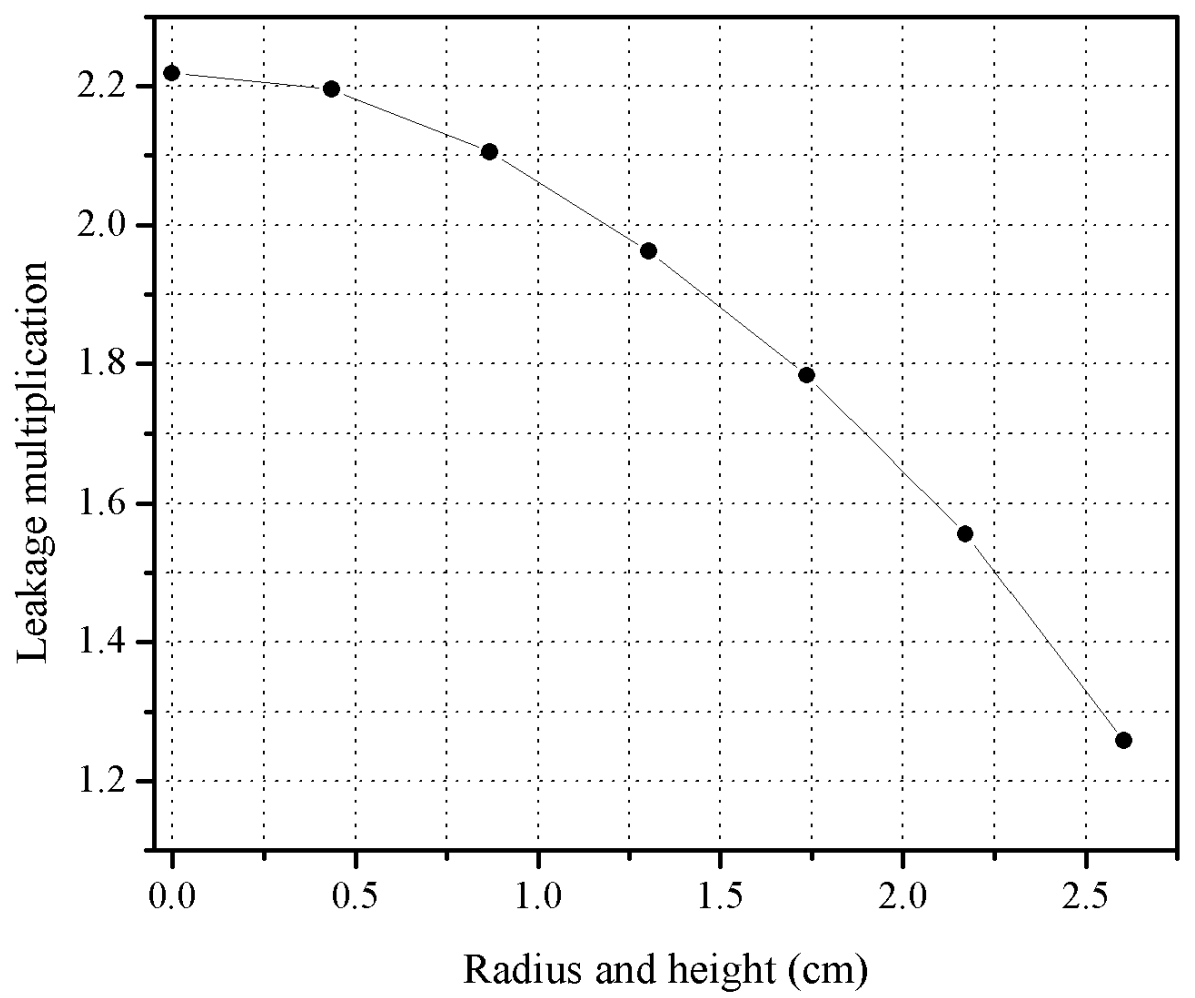

Fig. 1. Leakage multiplication vs. position for a 2-kg plutonium metal cylinder with diameter $=$ height. The plotted points are MCNPX results for positions along a straight line from the center of the cylinder to the top outer edge.

\section{Existing assay procedures}

It has long been known that kilogram-size plutonium metal samples produce large negative mass biases [6] when assayed with the point model multiplicity equations and that the cause of the biases is variable neutron multiplication in the samples. An empirical mass correction factor was added to the INCC code to account for the variablemultiplication bias. The final effective ${ }^{240} \mathrm{Pu}$ assay mass $\left(m_{240 e}\right)$ in the INCC code is 


$$
m_{240 e}=f_{M} m
$$

where $m$ is the effective ${ }^{240} \mathrm{Pu}$ assay mass from the point model equations and

$$
f_{M}=a_{M}+b_{M}(M-1)+c_{M}(M-1)^{2}
$$

where $a_{M}, b_{M}$, and $c_{M}$ are calibration parameters and $M$ is the neutron multiplication from the point model equations. The calibration parameters are obtained from measurements of plutonium metal samples with known effective ${ }^{240} \mathrm{Pu}$ masses. Recently, this correction factor has been improved [7] by the inclusion of another factor that depends on the alpha value from the point model equations. The final effective ${ }^{240} \mathrm{Pu}$ assay mass then becomes

$$
m_{240 e}=f_{M} f_{\alpha} m
$$

where

$$
f_{\alpha}=1+a_{\alpha} \alpha+b_{\alpha} \alpha^{2}
$$

where $a_{\alpha}$ and $b_{\alpha}$ are calibration parameters and $\alpha$ is the alpha value from the point model equations. These calibration parameters also are obtained from measurements of plutonium metal samples with known effective ${ }^{240} \mathrm{Pu}$ mass. The factor $f_{\alpha}$ is not presently in the INCC code.

\section{Purpose of this study}

The present study was undertaken to develop a physics-based, low-bias thermal neutron multiplicity assay procedure for impure plutonium metals. The empirical correction factors presently applied to remove the bias from variable multiplication are derived from particular sets of known plutonium samples and are not necessarily applicable to plutonium samples in general. These correction factors adjust the assay masses, but not the multiplication and alpha values; it is usually desirable to know the true $M$ and $\alpha$ values in addition to the true effective ${ }^{240} \mathrm{Pu}$ mass. It is also desirable to have a quantitative, physical basis for performing assay corrections. For these reasons, the point model multiplicity equations (hereafter referred to as the standard point model equations) were modified to become weighted point model equations, where weighting factors derived from Monte Carlo calculations are used to improve the assay values for the neutron multiplication, the alpha value, and the effective ${ }^{240} \mathrm{Pu}$ mass. This study was made possible by the recent MCNPX code, Version 2.5.B, which allows the use of spontaneous fission neutron sources and can tally the source and detected neutron multiplicity distributions. 


\section{WEIGHTED POINT MODEL EQUATIONS}

The standard point model multiplicity equations were modified as follows:

$$
\begin{aligned}
& S=m F_{0} \varepsilon v_{s 1} M(1+\alpha), \\
& D=\frac{1}{2} m F_{0} \varepsilon^{2} f_{d} v_{s} 2^{2}\left(f_{D}+\alpha f_{D}^{\alpha}\right), \\
& T=\frac{1}{6} m F_{0} \varepsilon^{3} f_{t} v_{s 3} M^{3}\left(f_{T}+\alpha f_{T}^{\alpha}\right),
\end{aligned}
$$

where

$$
\begin{aligned}
& f_{D}=w_{D}\left[1+c_{1}(M-1)\right], \\
& f_{D}^{\alpha}=w_{D}^{\alpha} c_{1}(M-1), \\
& f_{T}=w_{T}\left[1+c_{2}(M-1)+c_{3}(M-1)^{2}\right], \\
& f_{T}^{\alpha}=w_{T}^{\alpha}\left[c_{4}(M-1)+c_{3}(M-1)^{2}\right],
\end{aligned}
$$

where

$$
\begin{aligned}
& c_{1}=\frac{v_{s 1} v_{i 2}}{v_{s 2}\left(v_{i 1}-1\right)}, \\
& c_{2}=\frac{3 v_{s 2} v_{i 2}+v_{s 1} v_{i 3}}{v_{s 3}\left(v_{i 1}-1\right)}, \\
& c_{3}=\frac{3 v_{s 1} v_{i 2}^{2}}{v_{s 3}\left(v_{i 1}-1\right)^{2}}, \\
& c_{4}=\frac{v_{s 1} v_{i 3}}{v_{s 3}\left(v_{i 1}-1\right)},
\end{aligned}
$$

and where

$S, D, T=$ singles, doubles, and triples rates $(1 / \mathrm{s})$,

$$
m=\text { effective }{ }^{240} \mathrm{Pu} \text { mass }(\mathrm{g}),
$$


$M=$ neutron multiplication,

$\alpha=$ ratio of $(\alpha, n)$ to spontaneous fission neutrons,

$F_{0}={ }^{240} \mathrm{Pu}$ spontaneous fissions per gram ${ }^{240} \mathrm{Pu}$ per second,

$\varepsilon=$ neutron detection efficiency,

$f_{d}, f_{t}=$ doubles and triples gate fractions,

$v_{s l}, v_{s 2}, v_{s 3}=1 \mathrm{st}, 2 \mathrm{nd}$, and $3 \mathrm{rd}$ factorial moments of the ${ }^{240} \mathrm{Pu}$ spontaneous fission neutron multiplicity distribution,

$v_{i l}, v_{i 2}, v_{i 3}=1 \mathrm{st}, 2 \mathrm{nd}$, and 3 rd factorial moments of the neutron multiplicity distribution for the neutron induced fission of ${ }^{239} \mathrm{Pu}$,

and where $w_{D}, w_{D}^{\alpha}, w_{T}$, and $w_{T}^{\alpha}$ are the variable-multiplication weighting factors. The spontaneous fission and $(\alpha, n)$ contributions to the doubles and triples rates are all different functions of the multiplication and therefore have different weighting factors. The singles rate is proportional to the multiplication and so does not need weighting factors. When the four weighting factors are set to unity, the weighted point model equations become the standard point model equations.

The weighted point model equations are solved by eliminating $m$ and $\alpha$ from the equations for $S, D$, and $T$ to produce the quintic equation for the multiplication:

$$
k_{5} M^{5}+k_{4} M^{4}+k_{3} M^{3}+k_{2} M^{2}+k_{1} M+k_{0}=0,
$$

where

$$
\begin{aligned}
& k_{0}= 6 f_{d} T v_{s 1}\left(v_{i 1}-1\right)^{2}\left\{w_{D}\left[v_{s 2}\left(v_{i 1}-1\right)-v_{s 1} v_{i 2}\right]+w_{D}^{\alpha} v_{s 1} v_{i 2}\right\}, \\
& k_{1}=v_{s 1}\left(v_{i 1}-1\right)\left[6\left(w_{D}-w_{D}^{\alpha}\right) f_{d} T v_{s 1} v_{i 2}\left(v_{i 1}-1\right)+2 \varepsilon f_{t} D\left\{w_{T}^{\alpha} v_{s 1}\left[3 v_{i 2}^{2}-v_{i 3}\left(v_{i 1}-1\right)\right]-\right.\right. \\
&\left.\left.w_{T}\left[v_{s 3}\left(v_{i 1}-1\right)^{2}-\left(3 v_{s 2} v_{i 2}+v_{s 1} v_{i 3}\right)\left(v_{i 1}-1\right)+3 v_{s 1} v_{i 2}^{2}\right]\right\}\right], \\
& k_{2}=\varepsilon v_{s 1}\left[\varepsilon f _ { d } f _ { t } S \left\{w_{D}^{\alpha} w_{T} v_{i 2}\left[\left(3 v_{s 2} v_{i 2}+v_{s 1} v_{i 3}\right)\left(v_{i 1}-1\right)-3 v_{s 1} v_{i 2}^{2}-v_{s 3}\left(v_{i 1}-1\right)^{2}\right]-\right.\right. \\
&\left.w_{D} w_{T}^{\alpha}\left[v_{s 1} v_{i 2} v_{i 3}\left(v_{i 1}-1\right)+3 v_{s 2} v_{i 2}^{2}\left(v_{i 1}-1\right)-v_{s 2} v_{i 3}\left(v_{i 1}-1\right)^{2}-3 v_{s 1} v_{i 2}^{3}\right]\right\}+ \\
& 2 f_{t} D\left(v_{i 1}-1\right)\left\{w_{T}^{\alpha} v_{s 1}\left[v_{i 3}\left(v_{i 1}-1\right)-6 v_{i 2}^{2}\right]-w_{T}\left[\left(3 v_{s 2} v_{i 2}+v_{s 1} v_{i 3}\right)\left(v_{i 1}-1\right)-\right.\right.
\end{aligned}
$$




$$
\begin{gathered}
\left.\left.\left.6 v_{s 1} v_{i 2}^{2}\right]\right\}\right], \\
k_{3}=\varepsilon f_{t} v_{s 1}\left\{\varepsilon f _ { d } S \left[w_{D}^{\alpha} w_{T} v_{i 2}\left[v_{s 3}\left(v_{i 1}-1\right)^{2}-2\left(3 v_{s 2} v_{i 2}+v_{s 1} v_{i 3}\right)\left(v_{i 1}-1\right)+9 v_{s 1} v_{i 2}^{2}\right]-\right.\right. \\
\left.w_{D} w_{T}^{\alpha}\left\{\left[v_{s 2}\left(v_{i 1}-1\right)-2 v_{s 1} v_{i 2}\right] v_{i 3}\left(v_{i 1}-1\right)+3 v_{i 2}^{2}\left[3 v_{s 1} v_{i 2}-2 v_{s 2}\left(v_{i 1}-1\right)\right]\right\}\right]+ \\
\left.6\left(w_{T}^{\alpha}-w_{T}\right) D v_{s 1} v_{i 2}^{2}\left(v_{i 1}-1\right)\right\}, \\
k_{4}=\left(w_{D}^{\alpha} w_{T}-w_{D} w_{T}^{\alpha}\right) \varepsilon^{2} f_{d} f_{t} S v_{s 1} v_{i 2}\left[\left(3 v_{s 2} v_{i 2}+v_{s 1} v_{i 3}\right)\left(v_{i 1}-1\right)-9 v_{s 1} v_{i 2}^{2}\right],
\end{gathered}
$$

and

$$
k_{5}=3\left(w_{D}^{\alpha} w_{T}-w_{D} w_{T}^{\alpha}\right) \varepsilon^{2} f_{d} f_{t} S v_{s 1}^{2} v_{i 2}^{3}
$$

This equation is solved for $M$ by iteration. Then $\alpha$ and $m$ are obtained from

$$
\alpha=\frac{\varepsilon f_{d} S w_{D} M\left[v_{s 2}\left(v_{i 1}-1\right)+v_{s 1} v_{i 2}(M-1)\right]-2 D v_{s 1}\left(v_{i 1}-1\right)}{2 D v_{s 1}\left(v_{i 1}-1\right)-\varepsilon f_{d} S w_{D}^{\alpha} v_{s 1} v_{i 2} M(M-1)}
$$

and

$$
m=\frac{S}{F_{0} \varepsilon v_{s 1} M(1+\alpha)} \text {. }
$$

\section{BIAS SYSTEMATICS}

To study the systematics of the variable-multiplication bias, a series of Monte Carlo calculations was performed using the code MCNPX, Version 2.5.B, which can calculate the reduced factorial moments of the source and detected neutron multiplicity distributions and thus can be used to calculate the measured singles, doubles, and triples rates for the modeled experiment.

Calculations were done for cylindrical plutonium metal samples of $0.25,0.5,1,2,3$, and $4 \mathrm{~kg}$. The density was chosen as $18 \mathrm{~g} / \mathrm{cm}^{3}$, and the isotopic composition was chosen as $6 \%{ }^{240} \mathrm{Pu}$ and $94 \%{ }^{239} \mathrm{Pu}$ (atom percent). Default cross section libraries were used for all runs. For each sample mass, a series of 11 runs was done with different height/diameter ratios for the cylinders. The largest diameter was set to $15 \mathrm{~cm}$, and the maximum height was set to $30 \mathrm{~cm}$. One of the 11 runs for each mass was done with diameter $=$ height. Five runs were done with diameter $>$ height (pancakes) and five with diameter $<$ height (rods). For the pancake runs, the diameters were selected in geometric progression from diameter $=$ height to maximum diameter; likewise, for the rod runs the heights were 
chosen in geometric progression from diameter $=$ height to maximum height. For each sample mass and geometry two runs were done: one with a ${ }^{240} \mathrm{Pu}$ spontaneous fission source and one with an $(\alpha, n)$ source that had a ${ }^{240} \mathrm{Pu}$ spontaneous fission neutron energy spectrum. The source neutrons were always generated uniformly throughout the sample volume.

The neutron detector was set up in cylindrical geometry with the sample at the center. The detection material was pure ${ }^{3} \mathrm{He}$ with density $10 \mathrm{~g} / \mathrm{cm}^{3}$. The inner and outer diameters of the ${ }^{3} \mathrm{He}$ layer were $20 \mathrm{~m}$ and $40 \mathrm{~m}$, respectively; the inner and outer heights were also $20 \mathrm{~m}$ and $40 \mathrm{~m}$, respectively. No cadmium liner was used. With this huge sample cavity and massive detector, the detection efficiency was essentially $100 \%$, and the change in neutron multiplication from reflected neutrons was negligible. This experimental arrangement allowed the variable-multiplication effects from the sample to be isolated from detector parameters and sample/detector interactions.

This series of calculations consisted of $6 \times 11 \times 2=132$ MCNPX runs. To reduce the manual labor involved, the runs were automated by creating the MCNPX source decks and running the MCNPX code with a custom C code. Run times were typically 20 to 60 min each with a single $2.4 \mathrm{GHz}$ Pentium processor; the counting-statistics error was less than $1 \%(1 \sigma)$ for all count rates for most runs and less than $2 \%(1 \sigma)$ for all count rates for all runs.

The 132 MCNPX results files were processed with another custom $\mathrm{C}$ code. The singles, doubles, and triples rates and the leakage multiplication were obtained from a results file. The count rates were calculated from the reduced factorial moments. Because the efficiency was $100 \%$, the leakage multiplication was calculated from the singles rate. The leakage multiplication rather than the net multiplication was selected as the parameter for further analysis because it is more closely related to experimental data. The doubles and triples rates were then calculated from the standard point model using the leakage multiplication from the results file and using unity for the efficiency and the gate fractions. Because $S, D$, and $T$ are proportional to the ${ }^{240} \mathrm{Pu}$ mass, the assay multiplication, alpha value, and weighting factors are independent of the ${ }^{240} \mathrm{Pu}$ mass in these calculations. The ${ }^{240} \mathrm{Pu}$ mass was always set to $1 \mathrm{~g}$ (because it is arbitrary), and alpha was always set to 1 (for convenience). The variable-multiplication weighting factors are the ratios of the MCNPX rates to the point-model rates.

The point model calculations require input values for the factorial moments of the ${ }^{240} \mathrm{Pu}$ spontaneous fission neutron distribution and of the induced fission neutron distribution. These moments were obtained directly from the MCNPX code by doing special calculations. The MCNPX code includes the source distribution and moments in the output file. To get the spontaneous fission moments, a calculation was done with a ${ }^{240} \mathrm{Pu}$ spontaneous fission source in a sample of negligible density; the source moments are then the spontaneous fission neutron moments. The induced fission moments are slightly sample dependent, so the moments were determined for a low-multiplication plutonium sample with a ${ }^{240} \mathrm{Pu}$ spontaneous fission source as a convenient reference point. To get the induced fission moments from MCNPX, an $(\alpha, n)$ source with a ${ }^{240} \mathrm{Pu}$ spontaneous 
fission neutron energy spectrum was used as the neutron source. If a neutron source with $\mathrm{a}^{239} \mathrm{Pu}(\mathrm{n}, \mathrm{f})$ neutron energy spectrum is used instead of the ${ }^{240} \mathrm{Pu}$ spontaneous fission neutron energy spectrum, then the second factorial source moment is about $2 \%$ higher. Table II shows the factorial moments used as default values in the INCC code and the moments used for the point model calculations with the MCNPX data. The default induced fission moments in the INCC code are those for ${ }^{239} \mathrm{Pu}$ fission by $2-\mathrm{MeV}$ neutrons.

Table II. Spontaneous and induced fission factorial moments for the INCC code and for the point model calculations with the MCNPX data.

\begin{tabular}{|c|c|c|}
\hline Moment & Value from INCC & Value from MCNPX \\
\hline$v_{\mathrm{s} 1}$ & 2.154 & 2.153 \\
\hline$v_{\mathrm{s} 2}$ & 3.789 & 3.808 \\
\hline$v_{\mathrm{s} 3}$ & 5.211 & 5.273 \\
\hline$v_{\mathrm{i} 1}$ & 3.163 & 3.168 \\
\hline$v_{\mathrm{i} 2}$ & 8.240 & 8.376 \\
\hline$v_{\mathrm{i} 3}$ & 17.321 & 17.882 \\
\hline
\end{tabular}

Using the factorial moments from the last column of Table II, the variable-multiplication weighting factors were calculated with MCNPX for the 132 cases described above. For example, the triples weighting factor for a 2-kg plutonium sample with a spontaneous fission source is plotted vs. leakage multiplication in Fig. 2 for the 11 cylindrical configurations. The circles represent the pancakes, and the squares represent the rods. In this and most of the following plots, the errors are not plotted because they are generally smaller than the systematic variations. The interesting feature of this plot is that the weighting factor is insensitive to the sample geometry, so that the leakage multiplication approximately determines the weighting factor for all configurations. For a specified leakage multiplication, the pancake geometry has a slightly higher weighting factor than the rod geometry. This general behavior is similar for all the plutonium masses studied and for spontaneous fission and $(\alpha, n)$ sources. Furthermore, for a specified multiplication, the weighting factor is insensitive to the sample mass. This suggests that universal calibration curves of weighting factors vs. leakage multiplication can be used to obtain approximate assay masses with the weighted point model equations. 


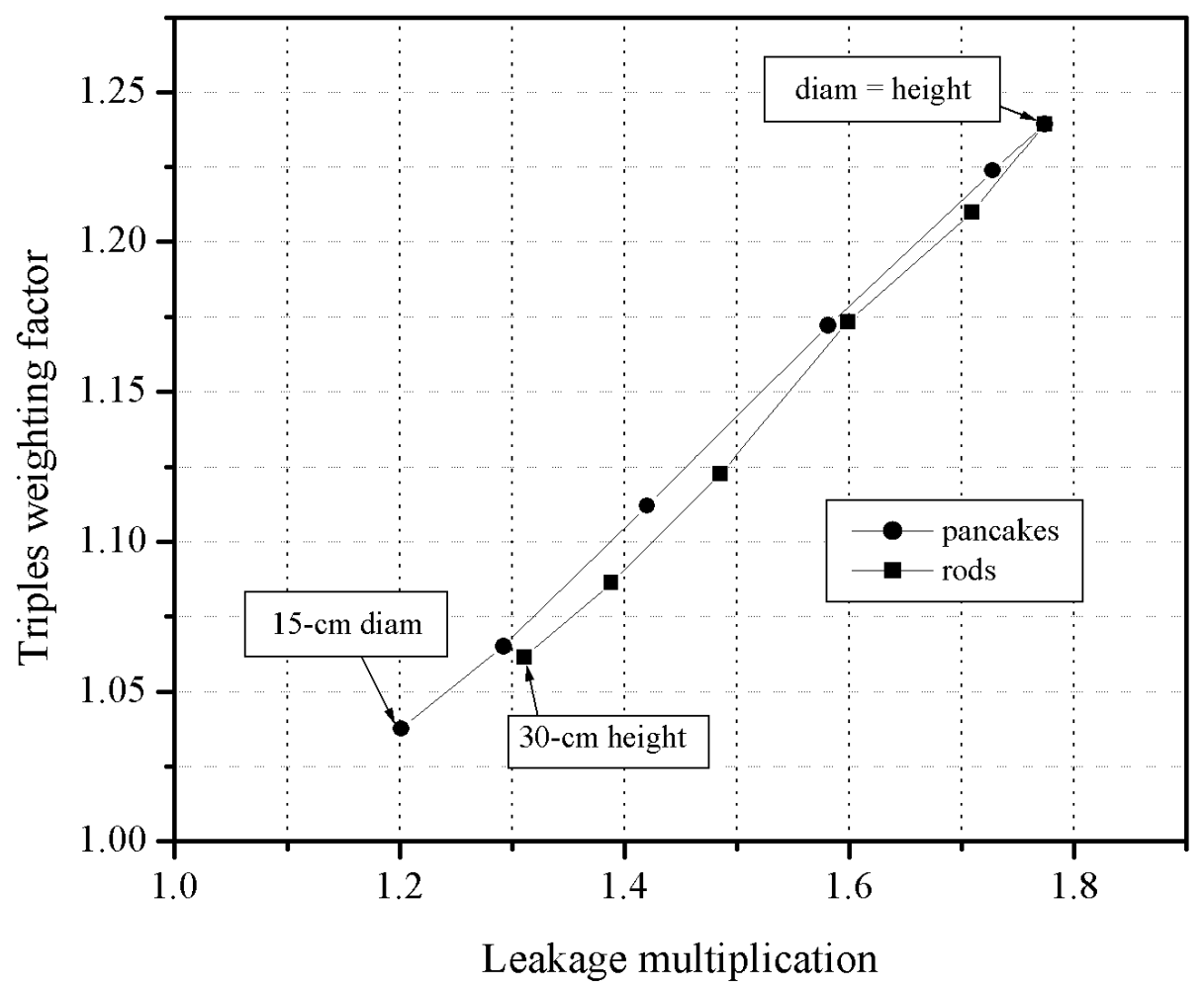

Fig. 2. Triples weighting factor vs. leakage multiplication for a 2-kg plutonium metal cylinder with various height/diameter ratios. The maximum diameter is $15 \mathrm{~cm}$, and the maximum height is $30 \mathrm{~cm}$.

Fig. 3 shows the triples weighting factor vs. leakage multiplication for all masses and geometries for a spontaneous fission source. The calibration curve shown is an unweighted least squares fit to the 66 data points. Numerically,

$$
w_{T}=1+0.3035(M-1)^{1.198}
$$

where $M$ is the leakage multiplication. The functional form was chosen simply to provide a good fit with few parameters and has no direct physical significance. 


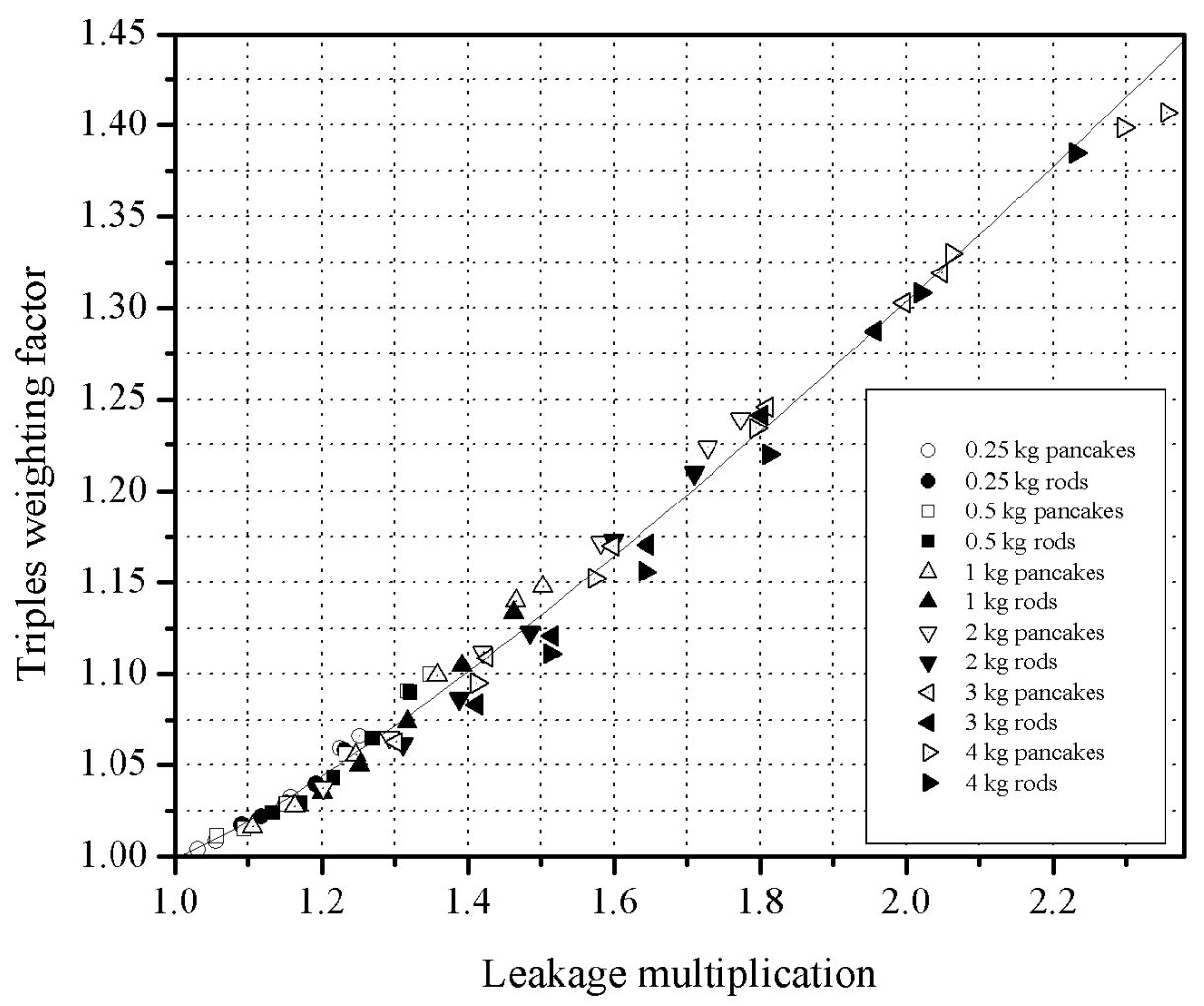

Fig. 3. Triples weighting factor vs. leakage multiplication for plutonium metal cylinders with a ${ }^{240} P u$ spontaneous fission source and masses from 0.25 to $4 \mathrm{~kg}$. Eleven height/diameter ratios are plotted for each mass. The solid line is the unweighted least squares fit to the 66 data points.

Similarly, the other three weighting factors for the 66 sample configurations with their calibration curves are shown in Figs. 4-6. Numerically,

$$
\begin{aligned}
& w_{T}^{\alpha}=0.6766+0.3354 M, \\
& w_{D}=1+0.09426(M-1)^{1.376},
\end{aligned}
$$

and

$$
w_{D}^{\alpha}=0.8871+0.1184 M
$$




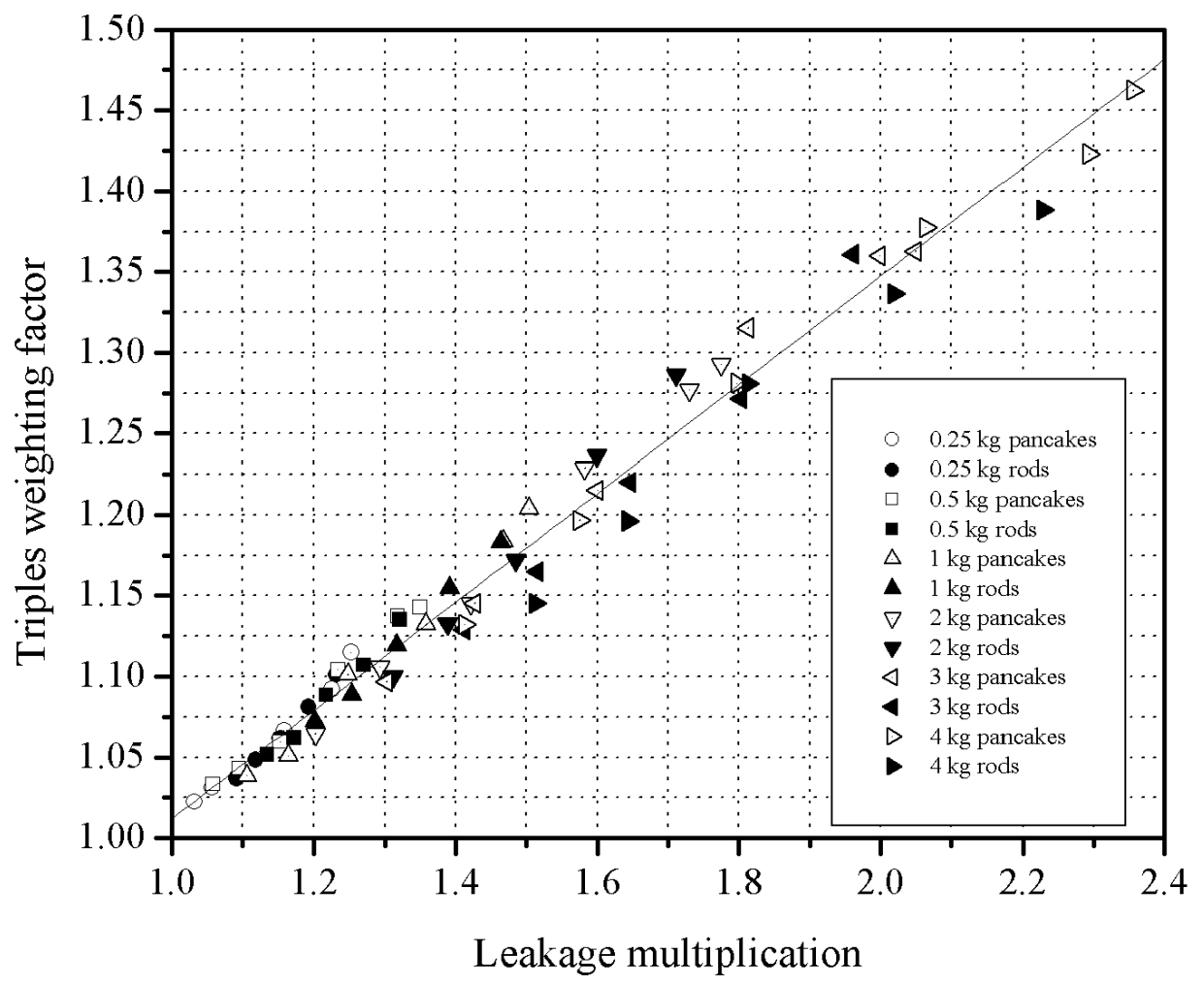

Fig. 4. Triples weighting factor vs. leakage multiplication for plutonium metal cylinders with masses from 0.25 to $4 \mathrm{~kg}$. The source is an $(\alpha, n)$ source with $a^{240} \mathrm{Pu}$ spontaneous fission neutron energy spectrum.

Eleven height/diameter ratios are plotted for each mass. The solid line is the unweighted least squares fit to the 66 data points. 


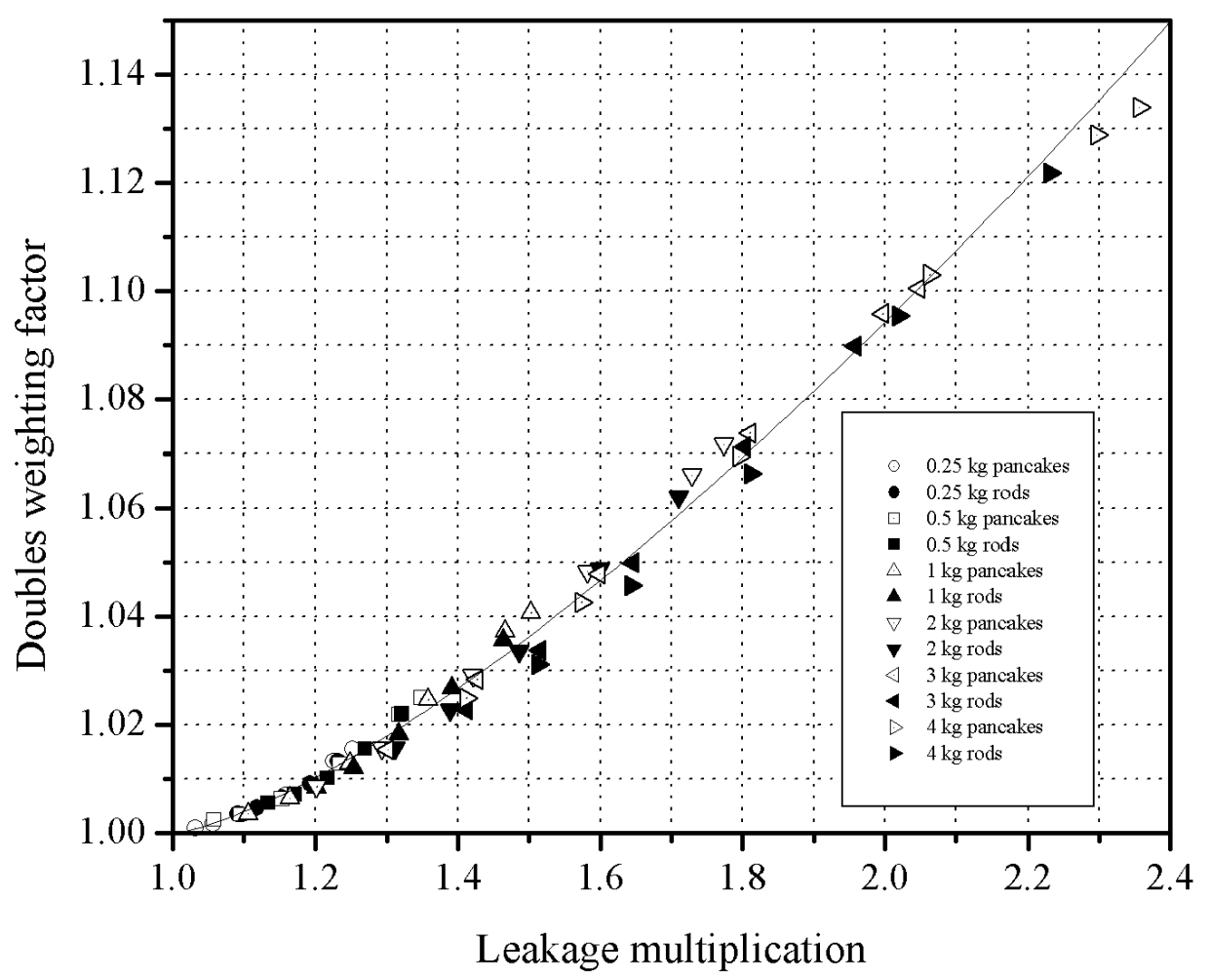

Fig. 5. Doubles weighting factor vs. leakage multiplication for plutonium metal cylinders with $a{ }^{240} P u$ spontaneous fission source and masses from 0.25 to $4 \mathrm{~kg}$. Eleven height/diameter ratios are plotted for each mass. The solid line is the unweighted least squares fit to the 66 data points. 


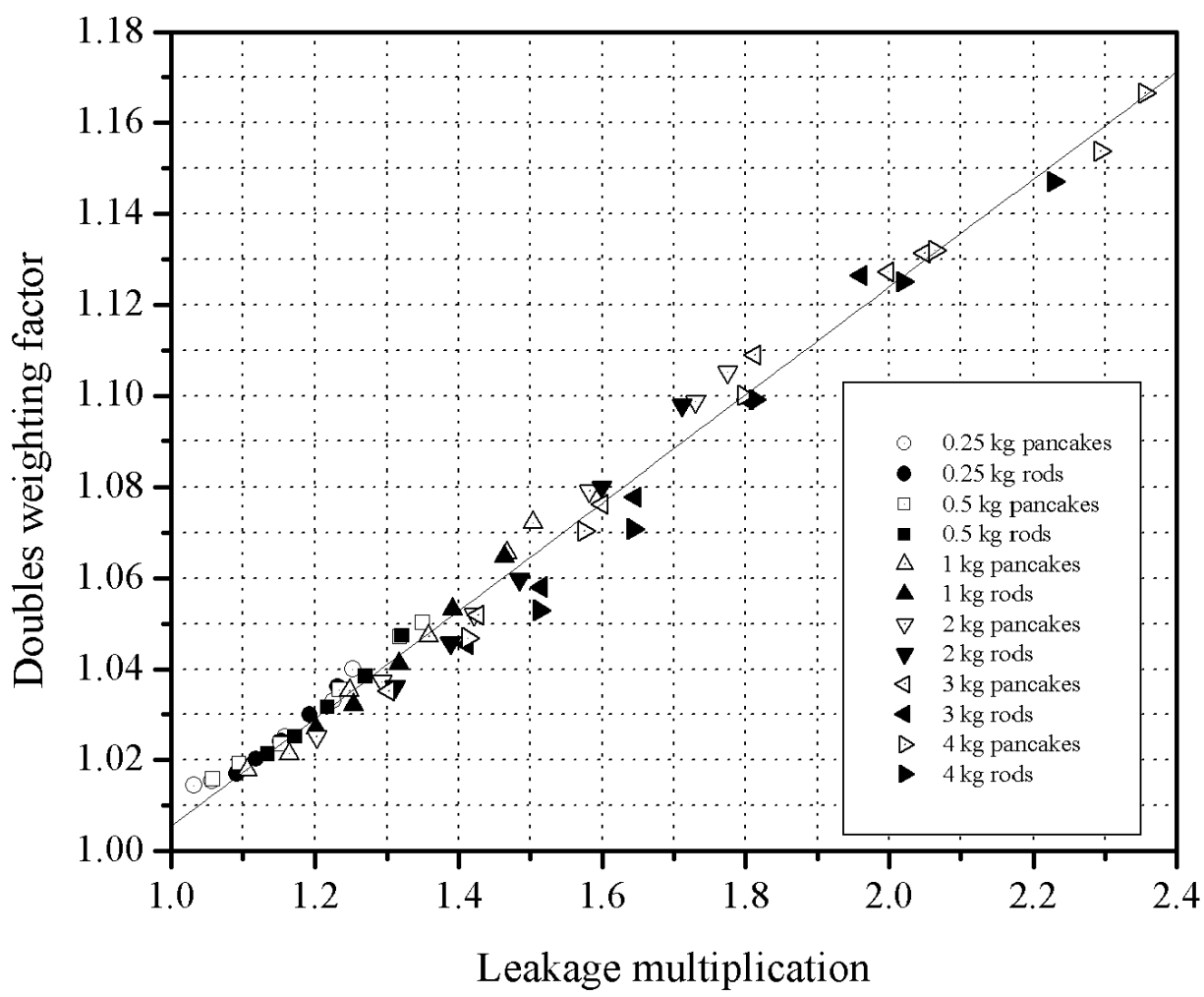

Fig. 6. Doubles weighting factor vs. leakage multiplication for plutonium metal cylinders with masses from 0.25 to $4 \mathrm{~kg}$. The source is an $(\alpha, n)$ source with $a^{240} \mathrm{Pu}$ spontaneous fission neutron energy spectrum. Eleven height/diameter ratios are plotted for each mass. The solid line is the unweighted least squares fit to the 66 data points.

\section{WEIGHTED POINT MODEL SIMULATED ASSAYS}

As a beginning exercise, the 66 sample configurations described in the previous section were used for simulated assays. The alpha values for the samples were arbitrarily all set to one. The singles, doubles, and triples rates for spontaneous fission and $(\alpha, n)$ sources were summed for each of the 66 samples. The summed rates were then used with the standard point model equations and the weighted point model equations to obtain simulated assay values for the multiplication, alpha, and ${ }^{240} \mathrm{Pu}$ mass for each sample. The weighting factor curves obtained in the previous section by least squares fitting were used for the weighted point model simulated assays.

Figs. 7-9 show the results for the ${ }^{240} \mathrm{Pu}$ mass, alpha, and multiplication, respectively. Fig. 7 shows the ratio of the assay to true ${ }^{240} \mathrm{Pu}$ mass vs. sample number. The sample plutonium masses and sample numbers are $0.25 \mathrm{~kg}$ (samples 1-11), $0.5 \mathrm{~kg}$ (samples $12-$ 22), $1 \mathrm{~kg}$ (samples 23-33), $2 \mathrm{~kg}$ (samples 34-44), $3 \mathrm{~kg}$ (samples 45-55), and $4 \mathrm{~kg}$ (samples 56-66). For each mass, the samples are ordered by increasing height/diameter ratio. The assay masses from the standard point model (plotted with circles) have large negative biases; e.g., the assay of the 4-kg sample with diameter $=$ height is biased 
negative by $61 \%$. For a given mass, higher multiplication corresponds to higher bias, so the thinnest pancake and the tallest rod have much lower biases than the sample with diameter $=$ height; this accounts for the oscillating character of the plotted points for the standard point model. The assay masses from the weighted point model (plotted with squares) are not highly biased because they use the weighting factors derived from the same 66 sample configurations. For a specified leakage multiplication, the correction factors have some variability as a result of the mass and geometry dependence, so the assays from the weighted point model also have some bias. The average mass ratio from the weighted point model is $1.0002 \pm 0.0017$.

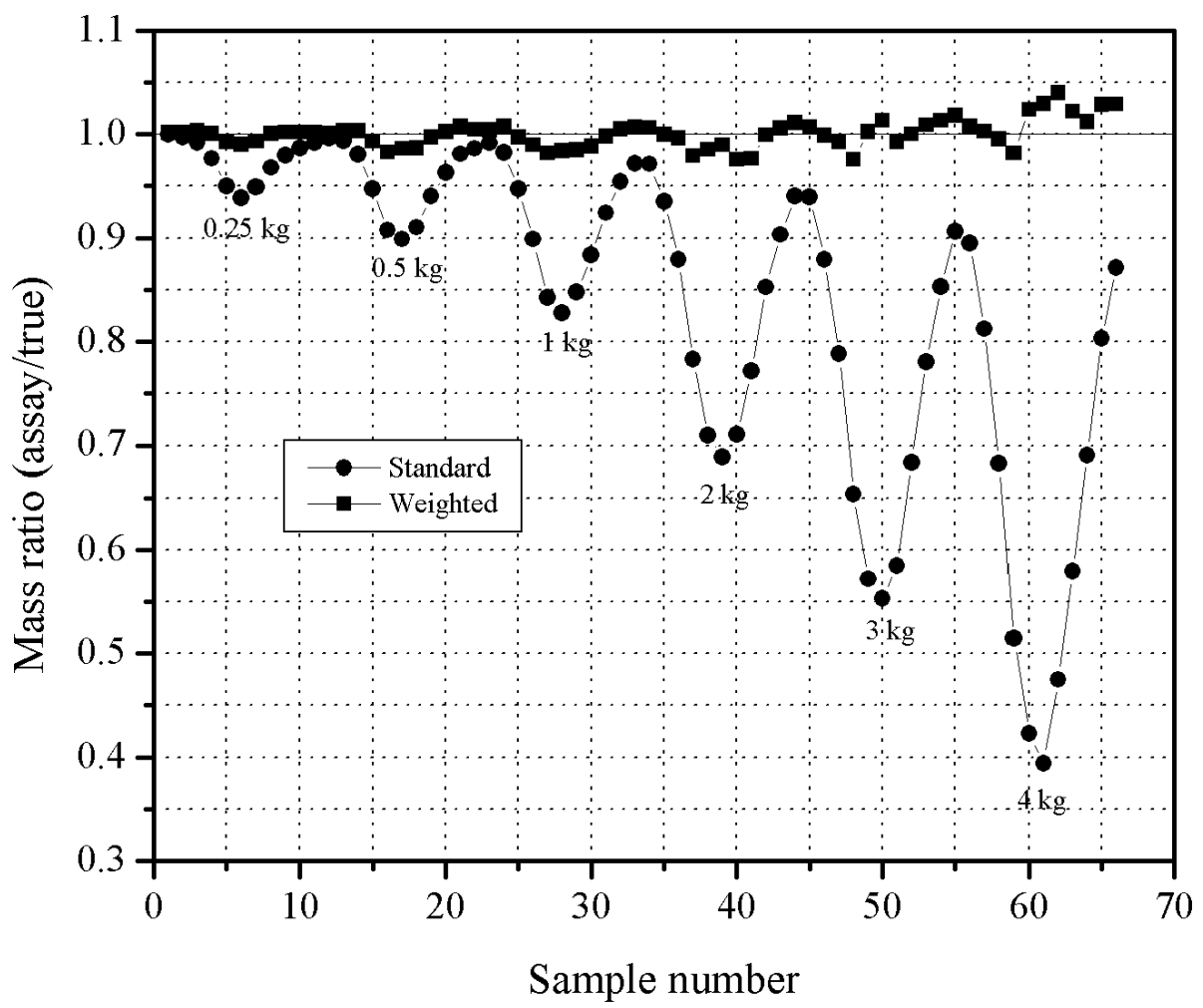

Fig. 7. Ratio of assay to true effective ${ }^{240} P u$ mass vs. sample number for the 66 plutonium cylinders used to determine the doubles and triples weighting factors. The assay masses are calculated with both the standard and weighted point models. The samples are ordered by increasing mass; for each mass the samples are ordered by increasing height/diameter ratio.

Fig. 8 shows the assay alpha values for the 66 samples for the standard and weighted models. The alpha value should be one for all samples. The alpha values are overestimated by the standard point model (circles), but are well determined by the weighted model (squares). The average alpha from the weighted model is $1.0000 \pm$ 0.0029 . This overestimation of alpha by the standard model also occurs when the true alpha is zero; therefore, a plutonium metal sample can appear to be highly impure when it is actually pure. 


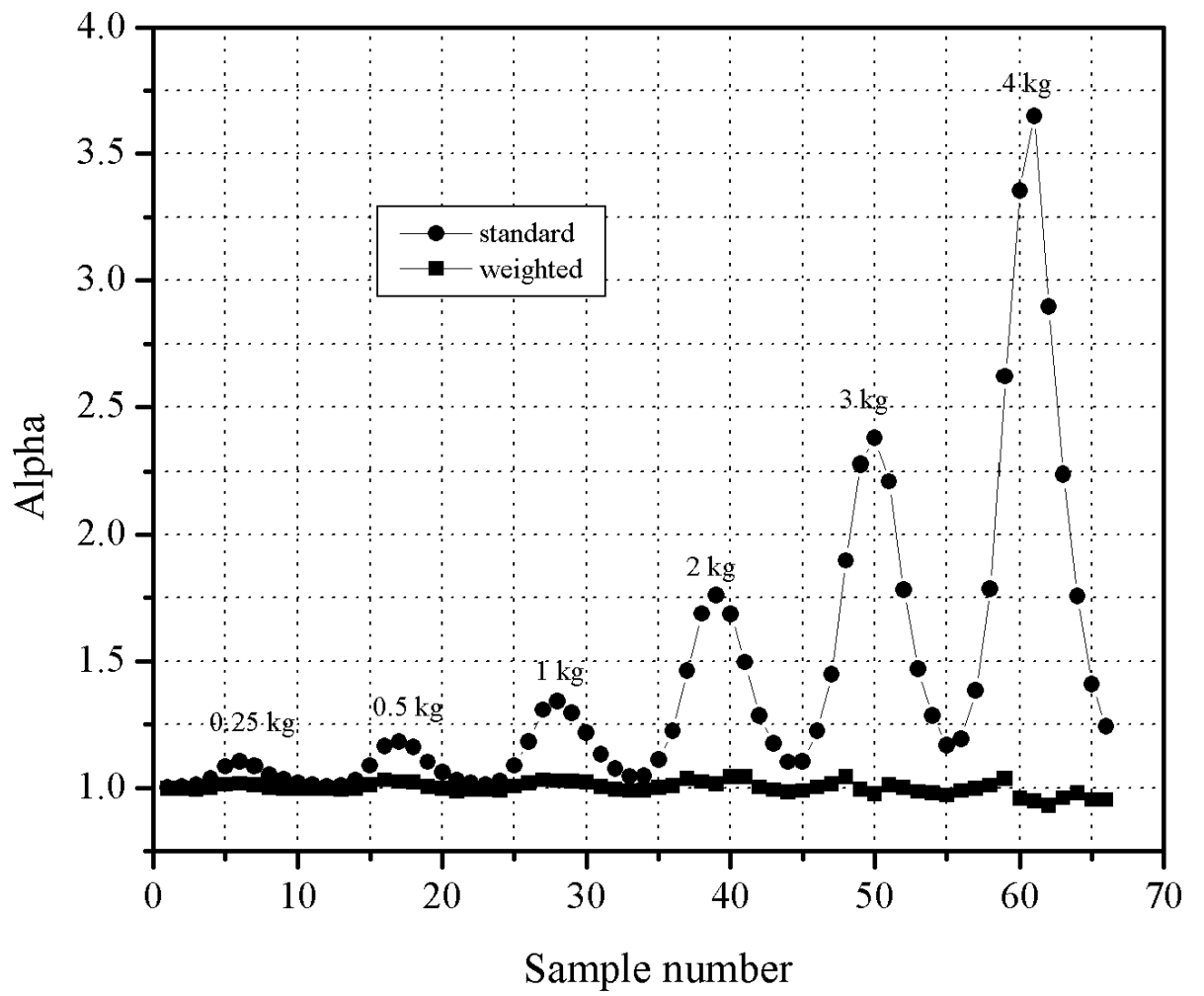

Fig. 8. Assay alpha value vs. sample number for the 66 plutonium cylinders used to determine the doubles and triples weighting factors. The alpha values are calculated with both the standard and weighted point models. The samples are ordered by increasing mass; for each mass, the samples are ordered by increasing height/diameter ratio.

Fig. 9 shows the ratio of the assay to true multiplication for the 66 samples for the standard and weighted models. Again, the standard model overestimates the true values, whereas the weighted model has little bias (ratio $=1.0000 \pm 0.0002)$. 


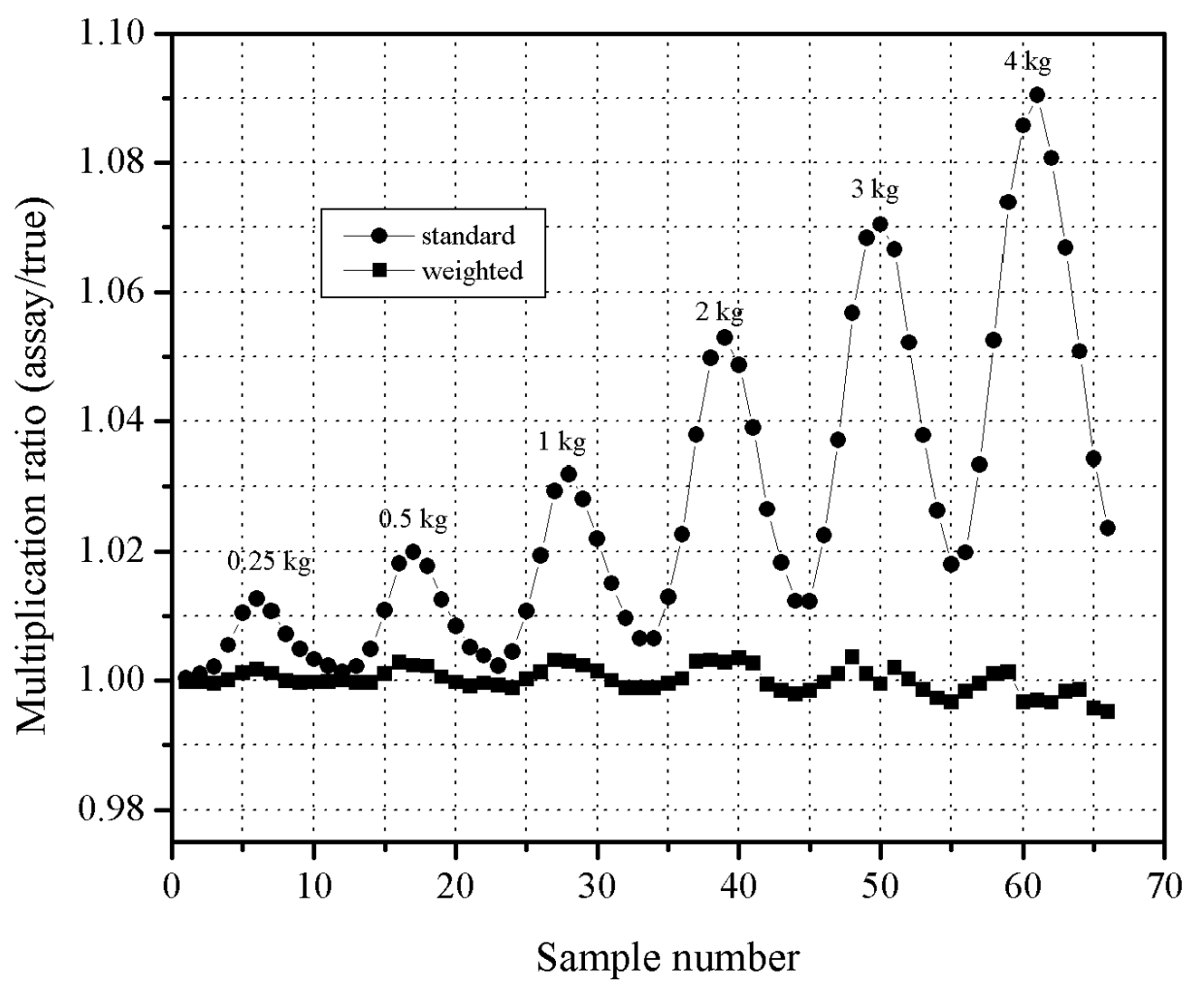

Fig. 9. Ratio of assay to true multiplication vs. sample number for the 66 plutonium cylinders used to determine the doubles and triples weighting factors. The multiplication values are calculated with both the standard and weighted point models. The samples are ordered by increasing mass; for each mass, the samples are ordered by increasing height/diameter ratio.

These calculations were repeated in a second exercise with a sample set different from the set used to determine the weighting factors. Using a random number generator, twenty-five plutonium metal assay samples were created with plutonium masses from 0.25 to $4 \mathrm{~kg}$, densities from 18 to $19 \mathrm{~g} / \mathrm{cm}^{3}$, alphas from 0 to 1 , and height/diameter ratios from $1 / 3$ to 3 . The isotopic composition remained $94 \%{ }^{239} \mathrm{Pu}$ and $6 \%{ }^{240} \mathrm{Pu}$ for all samples. Table III shows the sample data. 
Table III. Data for 25 randomized samples.

\begin{tabular}{|c|r|r|r|l|}
\hline $\begin{array}{c}\text { Sample } \\
\text { number }\end{array}$ & $\begin{array}{c}\text { Plutonium } \\
\text { mass }(\mathrm{g})\end{array}$ & $\begin{array}{c}\text { Density } \\
\left(\mathrm{g} / \mathrm{cm}^{3}\right)\end{array}$ & Height/diameter & Alpha \\
\hline 1 & 3636.3 & 18.189 & 1.0890 & 0.6368 \\
\hline 2 & 2353.8 & 18.018 & 0.8725 & 0.1180 \\
\hline 3 & 1464.6 & 18.247 & 0.3826 & 0.9464 \\
\hline 4 & 3145.1 & 18.927 & 0.7151 & 0.8983 \\
\hline 5 & 1411.2 & 18.338 & 2.6188 & 0.2405 \\
\hline 6 & 2566.0 & 18.560 & 2.8654 & 0.8403 \\
\hline 7 & 3673.6 & 18.794 & 2.3698 & 0.1293 \\
\hline 8 & 3002.7 & 18.533 & 1.8477 & 0.8894 \\
\hline 9 & 2012.3 & 18.174 & 1.5326 & 0.3299 \\
\hline 10 & 1570.1 & 18.152 & 0.3906 & 0.4243 \\
\hline 11 & 730.6 & 18.217 & 0.5328 & 0.9753 \\
\hline 12 & 761.5 & 18.920 & 0.6333 & 0.3779 \\
\hline 13 & 3994.4 & 18.916 & 0.6463 & 0.5475 \\
\hline 14 & 2691.3 & 18.180 & 2.3944 & 0.9836 \\
\hline 15 & 3748.3 & 18.716 & 0.3976 & 0.4364 \\
\hline 16 & 1932.7 & 18.871 & 0.8160 & 0.1462 \\
\hline 17 & 3723.1 & 18.835 & 1.0685 & 0.1234 \\
\hline 18 & 3197.4 & 18.421 & 0.7682 & 0.4383 \\
\hline 19 & 789.8 & 18.723 & 0.3903 & 0.9898 \\
\hline 20 & 3861.0 & 18.751 & 2.9009 & 0.7209 \\
\hline 21 & 2404.7 & 18.624 & 1.5506 & 0.7506 \\
\hline 22 & 1330.8 & 18.421 & 1.3701 & 0.3901 \\
\hline 23 & 1400.6 & 18.285 & 2.5927 & 0.5131 \\
\hline 24 & 2756.6 & 18.880 & 0.6326 & 0.2478 \\
\hline 25 & 1868.6 & 18.774 & 2.1370 & 0.4602 \\
\hline & & & & \\
\hline
\end{tabular}

MCNPX calculations were done for each of the 25 randomized samples. Both spontaneous fission and $(\alpha, n)$ sources were used for each sample. The singles, doubles, and triples rates for the $(\alpha, n)$ runs were weighted by the sample's alpha value and added to the corresponding rates for the spontaneous fission runs. As before, the efficiency and gate fractions were set to unity. The combined rates were then used with the standard and weighted point models to solve for multiplication, alpha, and ${ }^{240} \mathrm{Pu}$ mass.

The results are shown in Figs. 10-12. Fig. 10 shows the ratio of the assay to true multiplication vs. the true ${ }^{240} \mathrm{Pu}$ mass; the circles are data points for the standard point model, and the squares are data points for the weighted point model. Similarly, Fig. 11 shows the difference between the assay and true alpha values vs. the true ${ }^{240} \mathrm{Pu}$ mass, where the circles again are the data points for the standard point model. Finally, Fig. 12 shows the ratio of the assay to true mass vs. the true ${ }^{240} \mathrm{Pu}$ mass, where the circles and squares have the same meaning as above. The average ratio of the assay to true mass for the weighted model is $1.0029 \pm 0.0036$; the standard deviation of the 25 ratios is $1.8 \%$. This shows that the calibration curves for the weighting factors work well for a wide range of plutonium masses, alpha values, and cylindrical shapes. 


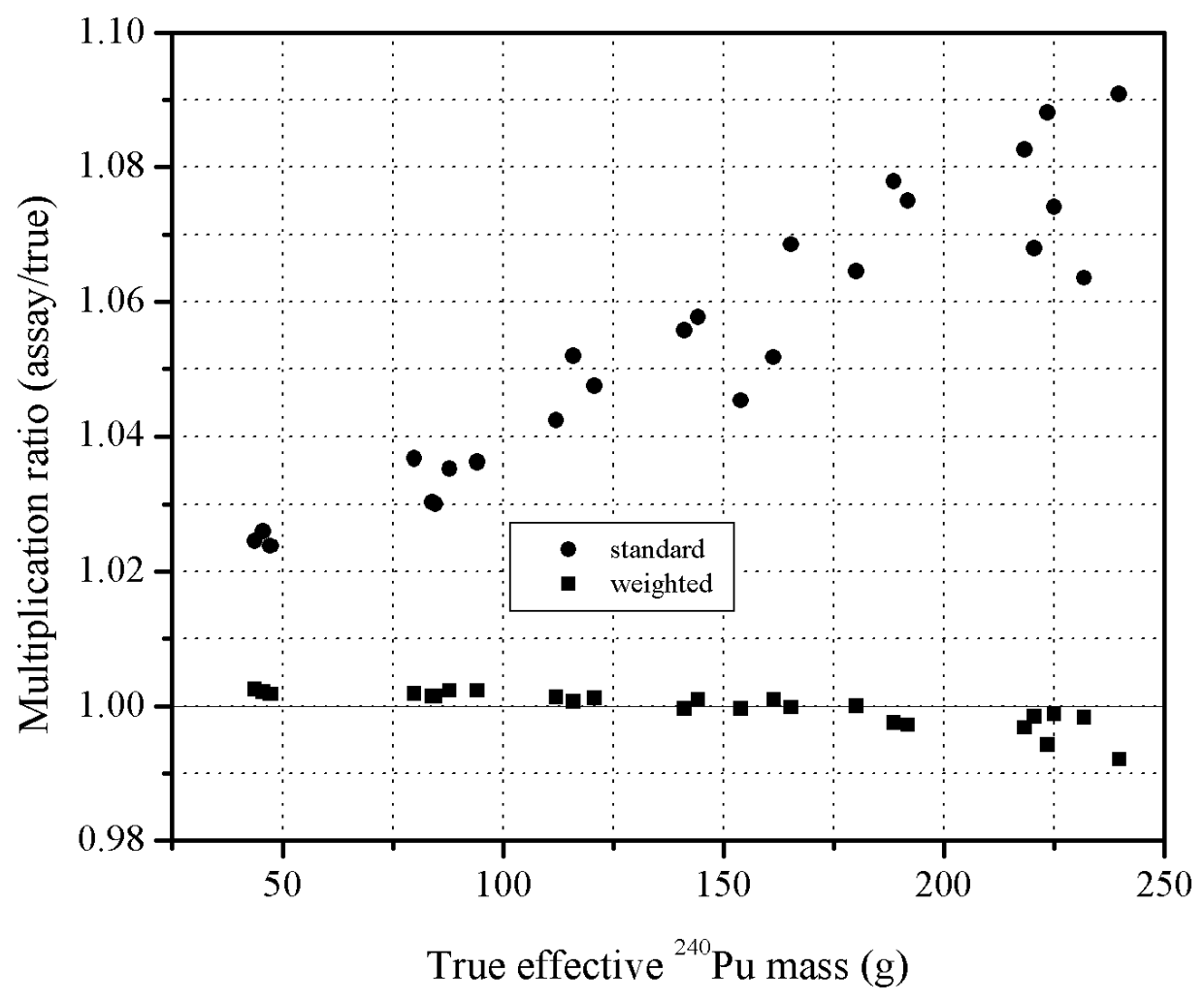

Fig. 10. Ratio of assay to true multiplication vs. true effective ${ }^{240} P$ u mass for 25 randomized plutonium metal samples. The calculations are done with both the standard and weighted point models. The points for the standard point model calculations show a lot of scatter in addition to the bias because of the randomized alpha values. 


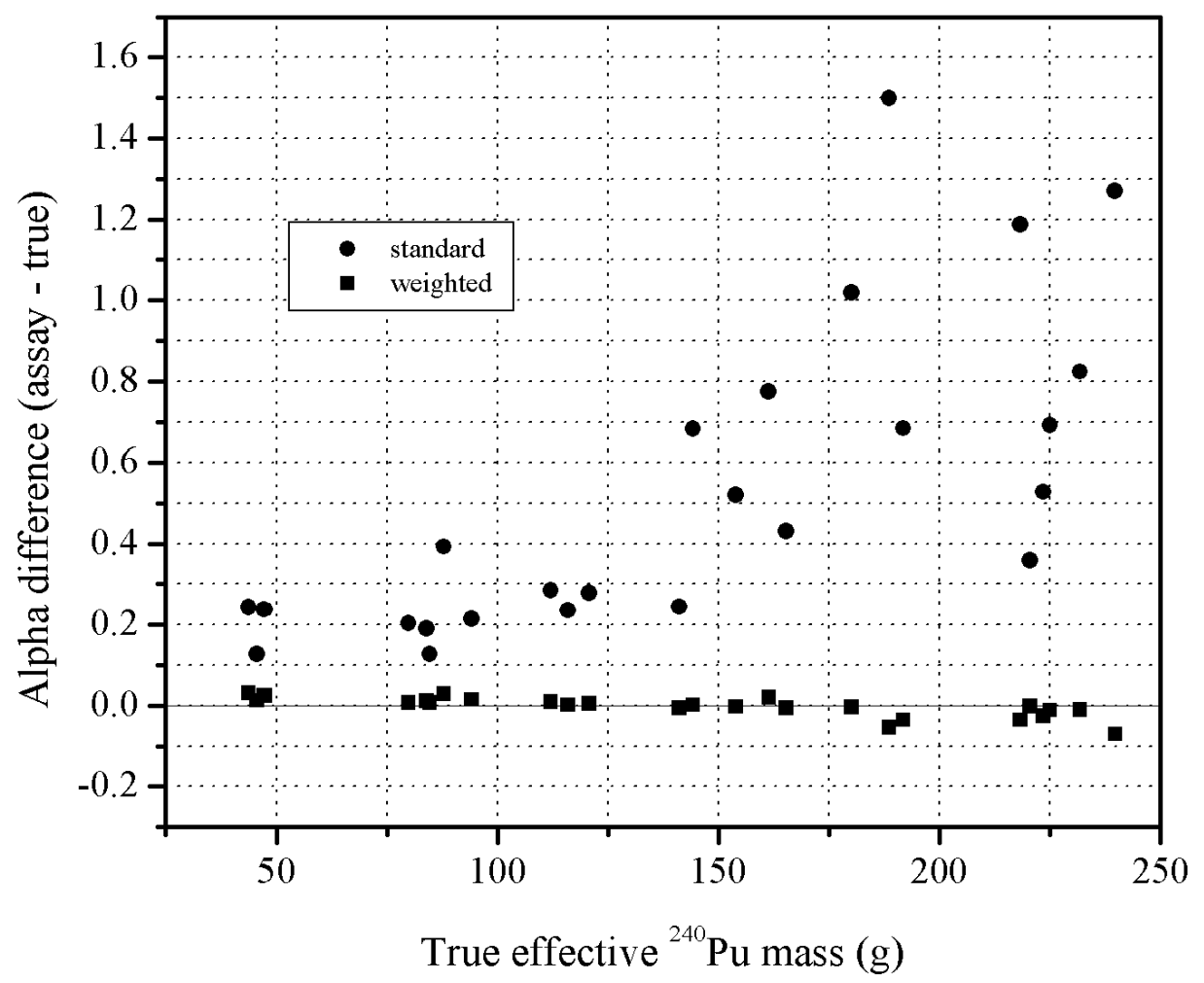

Fig. 11. Alpha value difference (assay minus true) vs. true effective ${ }^{240}$ Pu mass for 25 randomized plutonium metal samples. The calculations are done with both the standard and weighted point models. The points for the standard point model calculations show a lot of scatter in addition to the bias because of the randomized alpha values. 


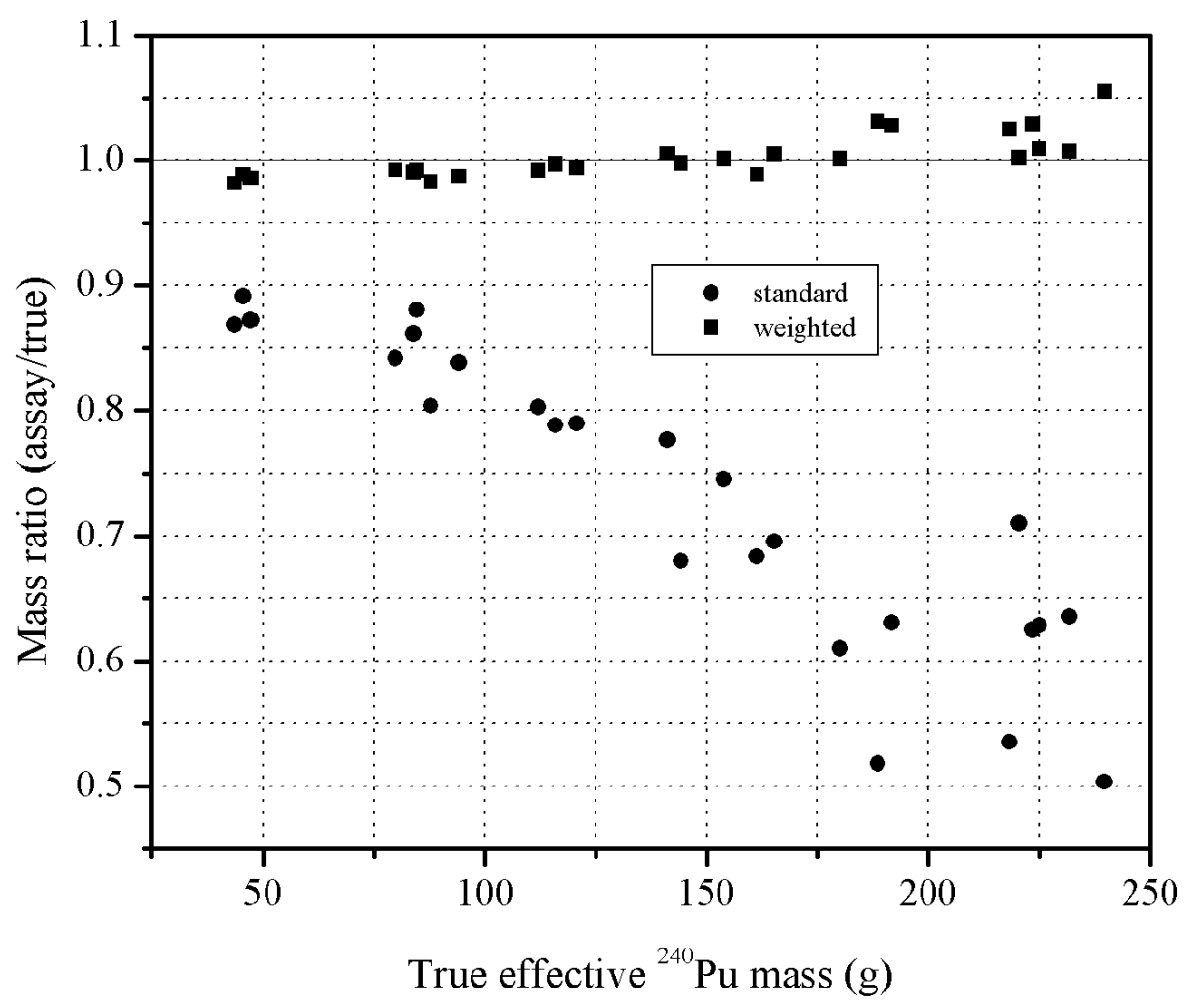

Fig. 12. Ratio of assay to true effective ${ }^{240} \mathrm{Pu}$ mass vs. true effective ${ }^{240} \mathrm{Pu}$ mass for 25 randomized plutonium metal samples. The calculations are done with both the standard and weighted point models. The points for the standard point model calculations show a lot of scatter in addition to the bias because of the randomized alpha values.

\section{SPECIAL ASSAY CONSIDERATIONS}

\section{Energy dependence}

Both the standard and weighted point models assume that the fission neutrons and the $(\alpha, n)$ neutrons have the same energy. Whereas the average fission neutron energy is approximately $2 \mathrm{MeV}$, the average $(\alpha, n)$ neutron energy can vary from about $0.4 \mathrm{MeV}$ to $4 \mathrm{MeV}$. Because the multiplication, induced-fission neutron moments, and detection efficiency (in real detectors) are energy dependent, the assay results will be energy dependent, and a bias can result from varying neutron energy. To study the energy dependence of the bias for one example, calculations were done with a 2-kg plutonium metal cylinder with diameter $=$ height, density $=18 \mathrm{~g} / \mathrm{cm}^{3}$, and an isotopic composition of $94 \%{ }^{239} \mathrm{Pu}$ and $6 \%{ }^{240} \mathrm{Pu}$. The alpha value was set to 0.5 . MCNPX runs were done for monoenergetic $(\alpha, n)$ neutron energies from 0.1 to $5 \mathrm{MeV}$. The calculational and analysis procedures were the same as described in the previous section. Fig. 13 shows the results for the standard and weighted point models; the ratio of the assay to true mass is plotted vs. the $(\alpha, n)$ neutron energy with circles for the standard-model data points and squares 
for the weighted-model data points. The relative energy dependences for the two models are very similar. The energy of the $(\alpha, n)$ neutrons can contribute significantly to the assay bias in both cases. In real multiplicity counters, the bias can be worse than shown in Fig. 13 because the neutron detection efficiency is also energy dependent.

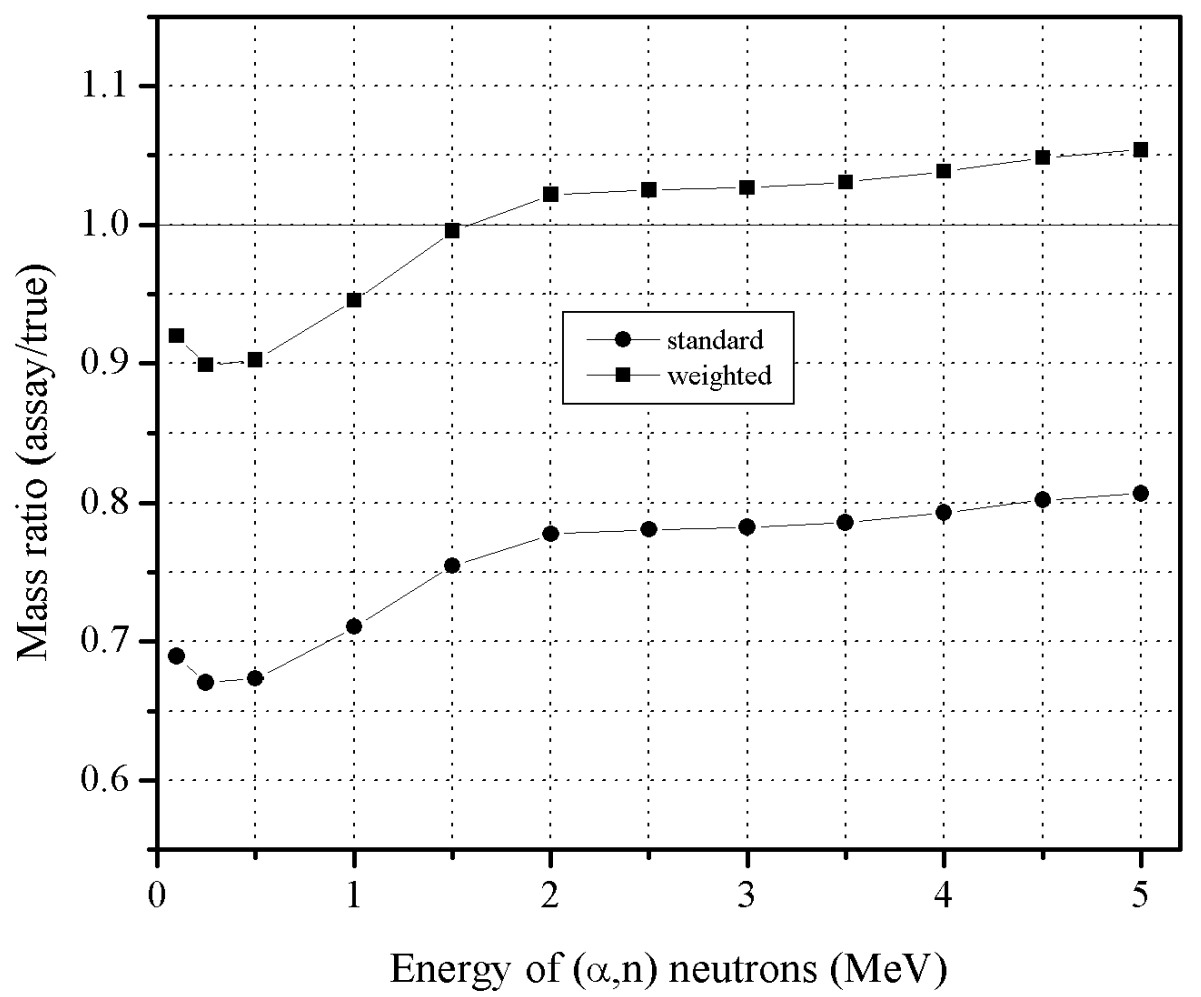

Fig. 13. Ratio of assay to true effective ${ }^{240}$ Pu mass vs. energy for a monoenergetic $(\alpha, n)$ neutron source. The sample is a 2-kg plutonium cylinder with diameter = height and with an alpha value of 0.5. The standard and weighted point models show very similar energy-dependent behavior.

Similar behavior has been observed previously [3] for the standard point model applied to the assay of plutonium oxide. In that study, the average energy of the $(\alpha, n)$ neutrons was estimated from the ring ratios, which are the ratios of the singles rate in the inner detector ring to the singles rate in the outer detector ring. The ring ratios primarily corresponded to neutron energies of $1-2 \mathrm{MeV}$, where the assay bias was small. The type of impurities in those oxide samples was unknown. If impurities found in plutonium metal samples produce $(\alpha, n)$ neutrons with energies of approximately $1-2 \mathrm{MeV}$, then the average energy-dependent bias will be small. Otherwise, large biases can occur. and the energy of the $(\alpha, n)$ neutrons will need to be taken into account in the assay of plutonium metals. 


\section{Detector sample cavity}

All of the MCNPX calculations described above were done with a very large sample cavity to isolate sample effects from detector effects. Another series of MCNPX calculations was done to study the variable-multiplication assay bias as a function of the cavity size. The sample used was the same 2-kg plutonium metal cylinder described above for the energy dependent calculations. For these calculations, the detection material was chosen as $90 \%$ hydrogen and $10 \%{ }^{3} \mathrm{He}$ (atom percent), and the detector cavity was lined with $0.0762 \mathrm{~cm}$ thick cadmium. The detection material was $10 \mathrm{~m}$ thick and had a density of $10 \mathrm{~g} / \mathrm{cm}^{3}$. The cavity diameter was always the same as the cavity height and the sample was placed at the center of the cavity. Cavity diameters of 1000, $300,50,30,20$, and $10 \mathrm{~cm}$ were studied. Fig. 14 shows the leakage multiplication and mass correction factor (ratio of true to assay mass) vs. the cavity diameter for the standard point model. The leakage multiplication increases as the cavity size decreases as a result of fissions induced by reflected neutrons. However, the mass correction factor is almost constant; although the overall sample multiplication increases, the distribution of multiplication values throughout the sample does not change enough to significantly affect the mass correction factor.

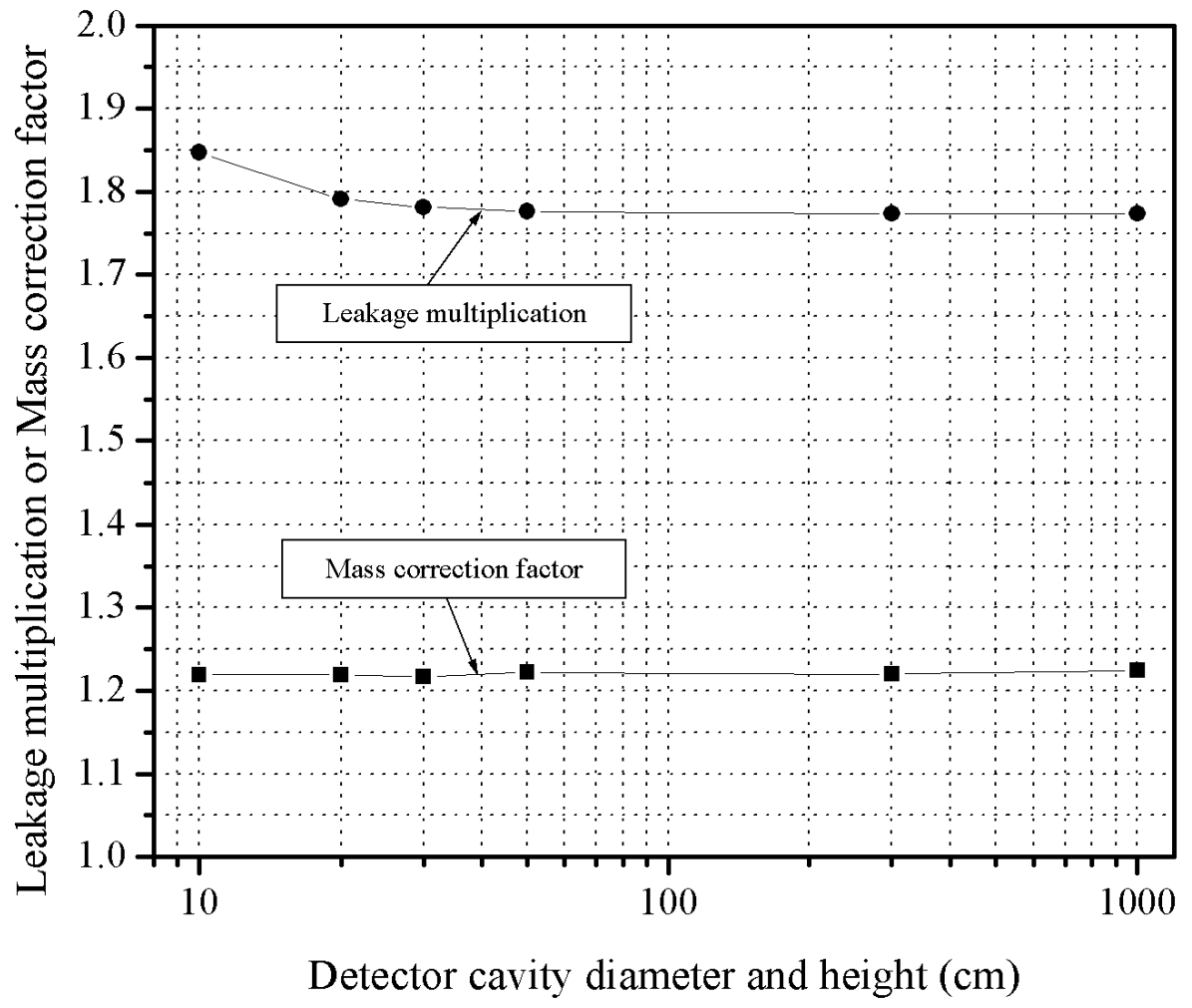

Fig. 14. Correction factors for the leakage multiplication and effective ${ }^{240} P u$ mass vs. detector cavity size for a 2-kg plutonium metal cylinder with diameter $=$ height centered in the cavity. 


\section{Sensitivity to detector parameters}

For highly multiplying plutonium metal samples, the assay masses are very sensitive to the detector parameters: efficiency, doubles gate fraction, and triples gate fraction. Consider the two sets of parameters shown in Table IV for the same detector. One set was derived from measurements of a ${ }^{252} \mathrm{Cf}$ source, which has a known neutron source strength. The other set was derived from measurements of impure plutonium oxide samples.

Table IV. Detector parameters derived from ${ }^{252} \mathrm{Cf}$ and plutonium.

\begin{tabular}{|c|c|c|}
\hline Parameter & Value from ${ }^{252} \mathrm{Cf}$ & $\begin{array}{c}\text { Value from } \\
\text { plutonium }\end{array}$ \\
\hline$\varepsilon$ & 0.5780 & 0.5743 \\
\hline$f_{d}$ & 0.4426 & 0.4480 \\
\hline$f_{t}$ & 0.1919 & 0.2030 \\
\hline
\end{tabular}

Assuming arbitrarily that the true parameters for the detector are those derived from the impure plutonium oxide samples, a series of calculations was done for a plutonium metal sample with a multiplication of 2 . The true singles, doubles, and triples rates were calculated from the standard point model for alpha values of $0,0.2,0.4,0.6,0.8$, and 1 . Then for each set of count rates the standard point model equations were solved for multiplication, alpha, and ${ }^{240} \mathrm{Pu}$ mass using the detector parameters derived from the ${ }^{252} \mathrm{Cf}$ measurements. The results are shown in Fig. 15, where the ratio of the assay to true mass is plotted vs. the assay alpha value. The use of the second parameter set results in a mass assay bias of $\sim 10 \%$ for true $\alpha=0$ up to a bias of $\sim 17 \%$ for true $\alpha=1$. The second parameter set also produces an overestimate of the alpha value; e.g., the pure plutonium metal sample appears to have an alpha of $\sim 0.1$. The sensitivity of the assay results to the detector parameters can complicate the comparison of the MCNPX and experimental results, because it is difficult to determine the true detector parameters with standard deviations below $1 \%$. 


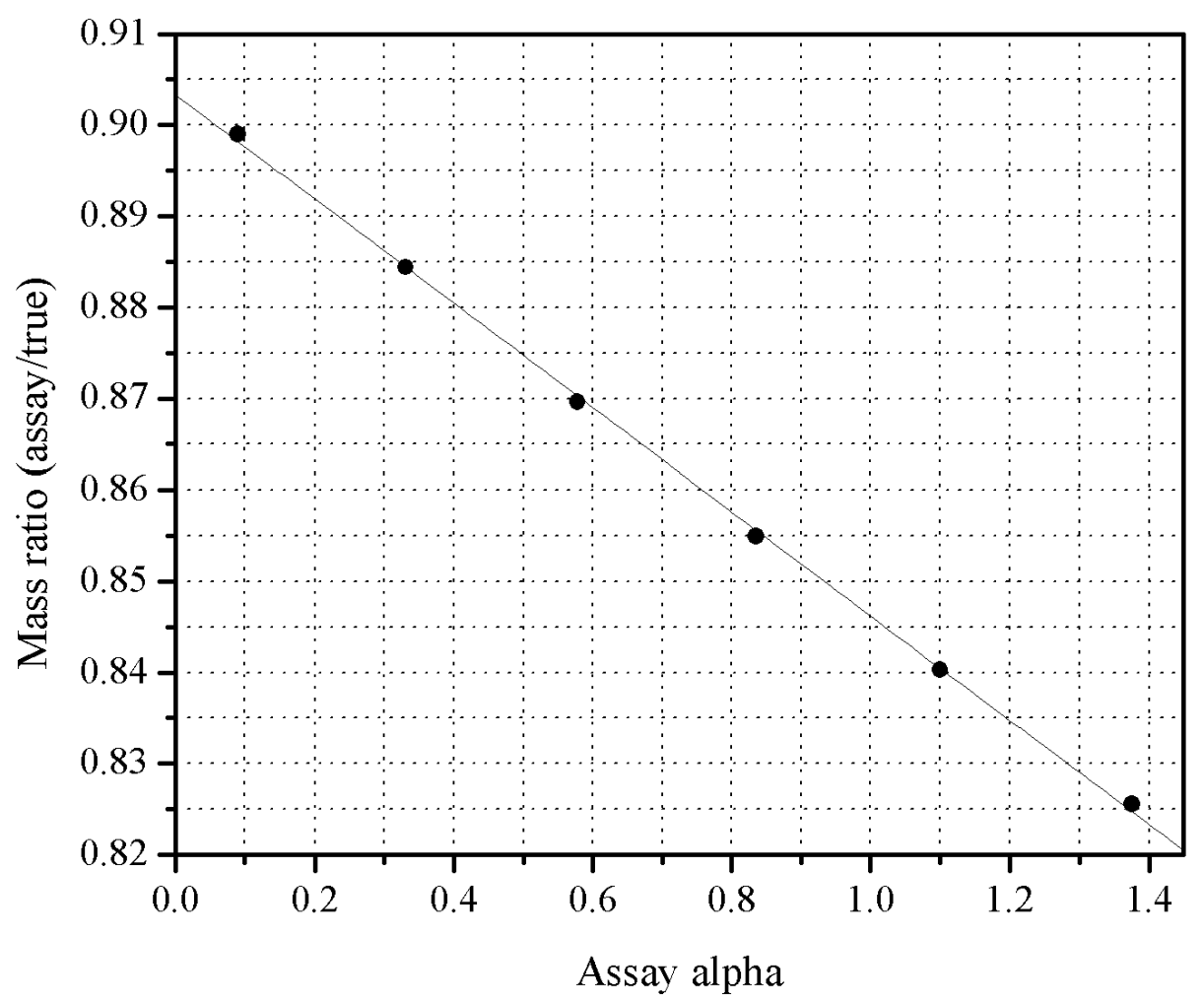

Fig. 15. Ratio of assay to true effective ${ }^{240} \mathrm{Pu}$ mass $v$ s. assay alpha value for a plutonium sample with a multiplication of 2 assayed with estimated detector parameters. Assays with the true detector parameters would produce a mass ratio of 1 for all alpha values.

\section{Other sample geometries}

The calibration curves for the weighting factors are based on MCNPX calculations for a series of masses and cylindrical geometries. When the geometry is not cylindrical, an additional bias can occur from the different multiplication profile in the sample. To study briefly one example of a different geometry, plutonium metal spheres with $\alpha=0$ and masses of 1, 2, and $3 \mathrm{~kg}$ were modeled with MCNPX. The mass correction factors obtained for each of these three spheres with a spontaneous fission source are shown in Fig. 16 together with the mass correction factors and least squares fit for the 66 samples used for the weighting factor calibrations. The mass corrections factors for the 1, 2, and 3 $\mathrm{kg}$ spheres fall below the least squares fit by $0.5 \%, 3.2 \%$, and $6.5 \%$, respectively. 


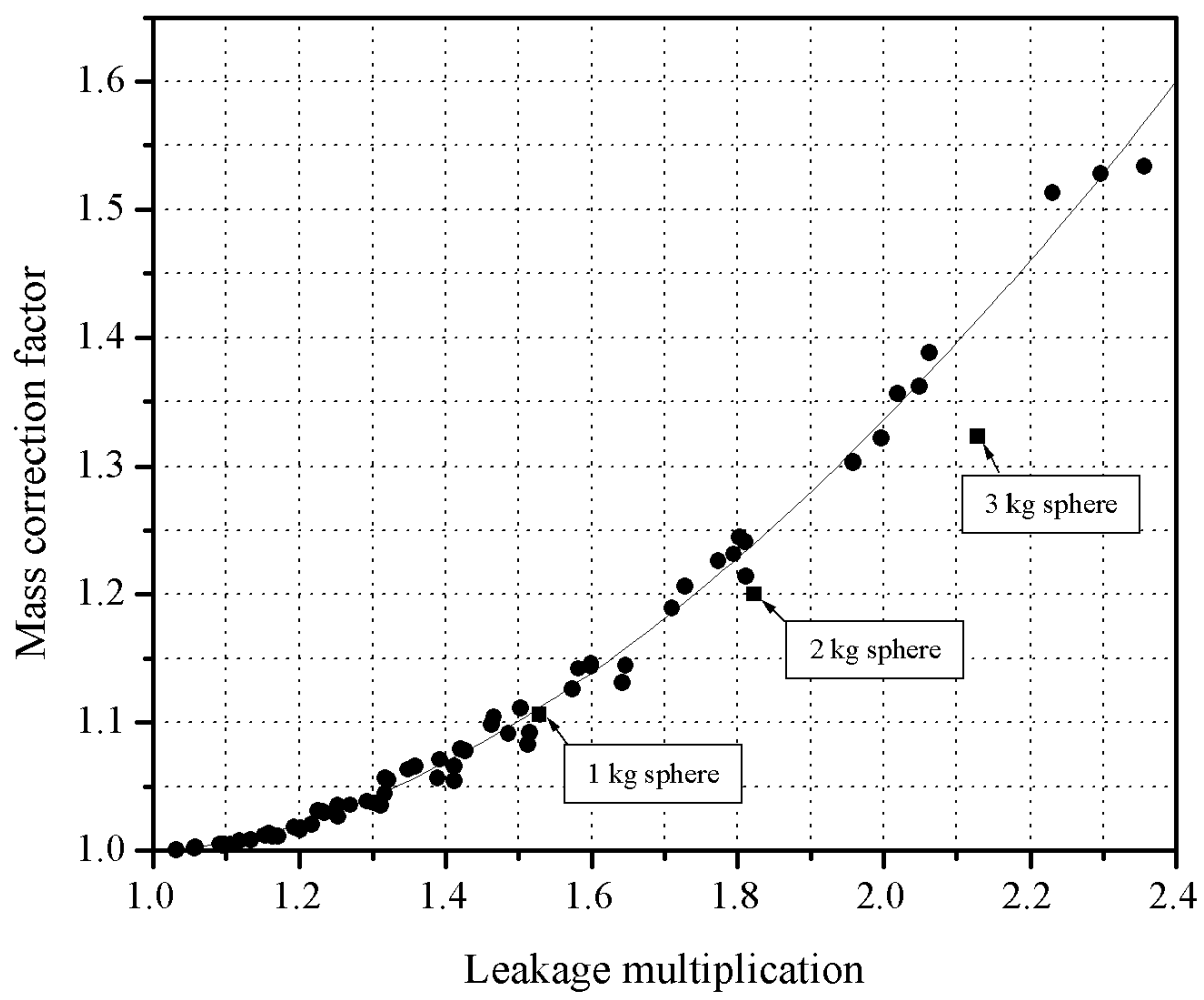

Fig. 16. Effective ${ }^{240} \mathrm{Pu}$ mass correction factor vs. leakage multiplication for the 66 plutonium metal cylinders used to determine the doubles and triples weighting factors and for plutonium metal spheres with masses of 1, 2, and $3 \mathrm{~kg}$. The neutron source is ${ }^{240} \mathrm{Pu}$ spontaneous fission. The spheres have the same density and isotopic composition as the cylinders. The curve is an unweighted least-squares fit to the 66 data points for the cylinders.

\section{Inhomogeneous samples}

All of the calculations described above in this report were done with the assumption that the samples are homogeneous. If the samples are inhomogeneous, assay biases can result. For example, if sample impurities are concentrated in the upper or lower regions of a sample, the multiplication distribution for the $(\alpha, n)$ neutrons will be different from the multiplication distribution for the spontaneous fission neutrons. In this case, the weighting factors will be inaccurate, and a bias will result. This effect was studied for the hypothetical case of a 2-kg plutonium cylinder with diameter $=$ height and with $\alpha=1$, where the $(\alpha, n)$ source filled a fraction $f$ (the fill fraction) of the volume such that the source was uniform across the diameter and uniform along the axis for the fraction $f$ of the length, starting at one end. The $(\alpha, n)$ source had a ${ }^{240} \mathrm{Pu}$ spontaneous fission neutron energy distribution. MCNPX calculations were done for values of $f$ from 0.1 to 1 . The resulting $S, D$, and $T$ rates were then solved for $M, \alpha$, and $m$ using the weighted point model equations with the weighting factors determined earlier in this report. The results are shown in Fig. 17, where the ratio of assay to true mass is plotted vs. the fill fraction. 
The effect of an inhomogeneous $(\alpha, n)$ source can be quite large when the $(\alpha, n)$ source is concentrated at one end of the cylinder; e.g., if $f=0.1$, then the bias is $-28 \%$ in the example studied. Fortunately, $\alpha<<1$ for most real plutonium metal items.

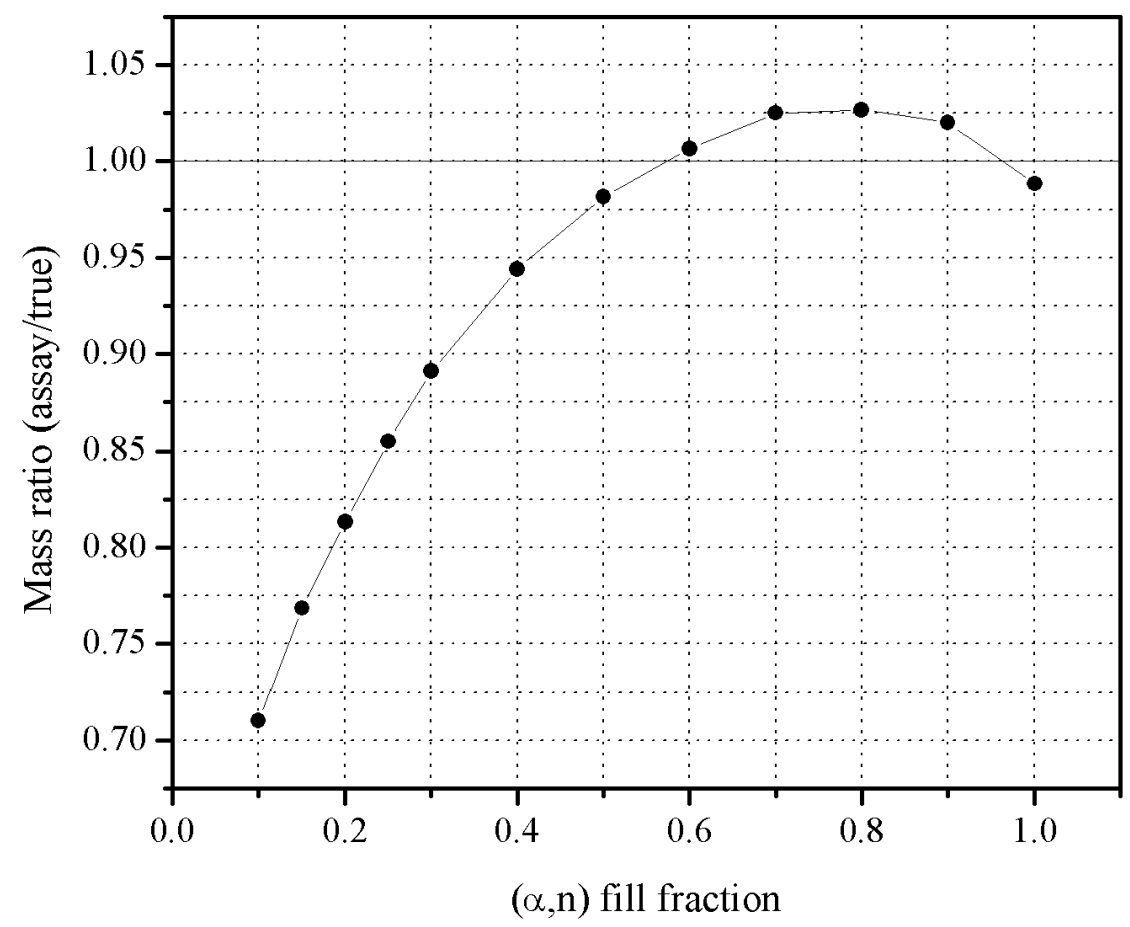

Fig. 17. Ratio of assay to true mass vs. $(\alpha, n)$ fill fraction $(f)$ for a 2-kg plutonium metal cylinder with diameter $=$ height, where the $(\alpha, n)$ source with a ${ }^{240} P u$ spontaneous fission neutron spectrum and with $\alpha=1$ fills the fraction $f$ of the cylinder along the axis, starting at one end. The assay calculation was done with the weighted point model equations.

\section{COMPARISON WITH EXPERIMENT}

\section{Comparison with measurement data from a plutonium oxide sample with known mass and isotopic composition}

A slightly impure plutonium oxide sample $(\mathrm{id}=\mathrm{PEO} 381)$ with an effective ${ }^{240} \mathrm{Pu}$ mass of $64.98 \mathrm{~g}$ was measured on $2000 \mathrm{Aug} 10$ (INCC results file $=08 \mathrm{AO} 4252$. VER) in a multiplicity counter $(\mathrm{id}=5 \mathrm{RMC})$ with the following established parameters:

$$
\begin{aligned}
& \varepsilon=0.535 \\
& f_{d}=0.6500 \\
& f_{t}=0.4225 .
\end{aligned}
$$

The measured count rates corrected for background and electronic deadtime were 


$$
\begin{aligned}
& S_{m}=65223 \pm 55 \mathrm{~s}^{-1} \\
& D_{m}=16480 \pm 29 \mathrm{~s}^{-1} \\
& T_{m}=5029 \pm 78 \mathrm{~s}^{-1} .
\end{aligned}
$$

Using the standard point model multiplicity equations and the default INCC factorial moments, these rates give the following assay results:

$$
\begin{aligned}
& M=1.079 \pm 0.002 \\
& \alpha=0.682 \pm 0.019 \\
& m=65.88 \pm 0.87 \mathrm{~g} .
\end{aligned}
$$

The assay to true ${ }^{240} \mathrm{Pu}$ mass ratio is thus $1.014 \pm 0.013$.

The PEO381 sample was modeled with MCNPX in the ideal detector that was used earlier for the bias systematics study and that has an efficiency of essentially $100 \%$. Calculations were done with ${ }^{240} \mathrm{Pu}$ spontaneous fission sources and with $(\alpha, \mathrm{n})$ sources that had a ${ }^{240} \mathrm{Pu}$ spontaneous fission neutron spectrum. The true multiplication and alpha value of the sample were initially unknown. The density of the sample was adjusted with a series of MCNPX runs so that the calculated multiplication agreed with the INCC assay value, which is known to be very accurate when the multiplication is low. The alpha value was then calculated from this multiplication, the known effective ${ }^{240} \mathrm{Pu}$ mass, and the measured singles rate:

$$
\alpha=\frac{S_{m}}{m F_{0} v_{s 1} M \varepsilon}-1=0.7056
$$

Using this alpha value, the known effective ${ }^{240} \mathrm{Pu}$ mass, the known detector parameters, and the MCNPX results, the simulated doubles and triples rates were calculated to be

$$
\begin{aligned}
& D_{M C N P X}=16512 \mathrm{~s}^{-1} \\
& T_{M C N P X}=5184 \mathrm{~s}^{-1} .
\end{aligned}
$$

The precisions of the MCNPX results are negligibly small compared with the experimental precisions. The MCNPX singles rate is the same as the experimental rate because of the alpha calculation above. The results are

$$
\frac{D_{M C N P X}}{D_{m}}=\frac{16512}{16480 \pm 29}=1.002 \pm 0.002
$$

and

$$
\frac{T_{M C N P X}}{T_{m}}=\frac{5184}{5029 \pm 78}=1.031 \pm 0.016
$$


The errors shown are for precision only. Additional error components come from the detector and nuclear parameters used in the calculations, energy-dependent effects, and the approximate modeling of PEO381. Therefore, measurement and calculation are in reasonable agreement for this sample.

\section{Mass correction factors}

All other measurement data used for comparison with the MCNPX calculations were obtained from the FB-Line Neutron Multiplicity Counter (FBLNMC) [8] at Savannah River.

Empirical mass correction factors for 232 plutonium metal samples were obtained as the ratios of the known plutonium masses to those calculated from the measured singles, doubles, and triples rates and measured isotopic compositions using the standard point model multiplicity equations with INCC default spontaneous fission and induced fission moments. The least-squares fit to the 232 correction factors vs. the measured neutron multiplication is

$$
f_{m}=a_{M}+b_{M}(M-1)+c_{M}(M-1)^{2},
$$

where

$$
\begin{aligned}
& f_{m}=\text { mass correction factor, } \\
& M=\text { measured neutron multiplication, } \\
& a_{M}=1, \\
& b_{M}=0.14607,
\end{aligned}
$$

and

$$
c_{M}=0.1485 \text {. }
$$

This equation is plotted in Fig. 18 with the corresponding calibration curve and data points obtained from the $66 \mathrm{MCNPX}$ samples. The agreement between the calculated and measured mass correction factors is not quite as good as it seems because the MCNPX results are for $\alpha=0$, whereas some of the experimental results are for impure samples with alpha values typically between 0.1 and 0.4 . Furthermore, the multiplication range for the 232 samples is approximately 1.2 to 1.6 compared with a range of 1.0 to 2.6 for the MCNPX calculations. 


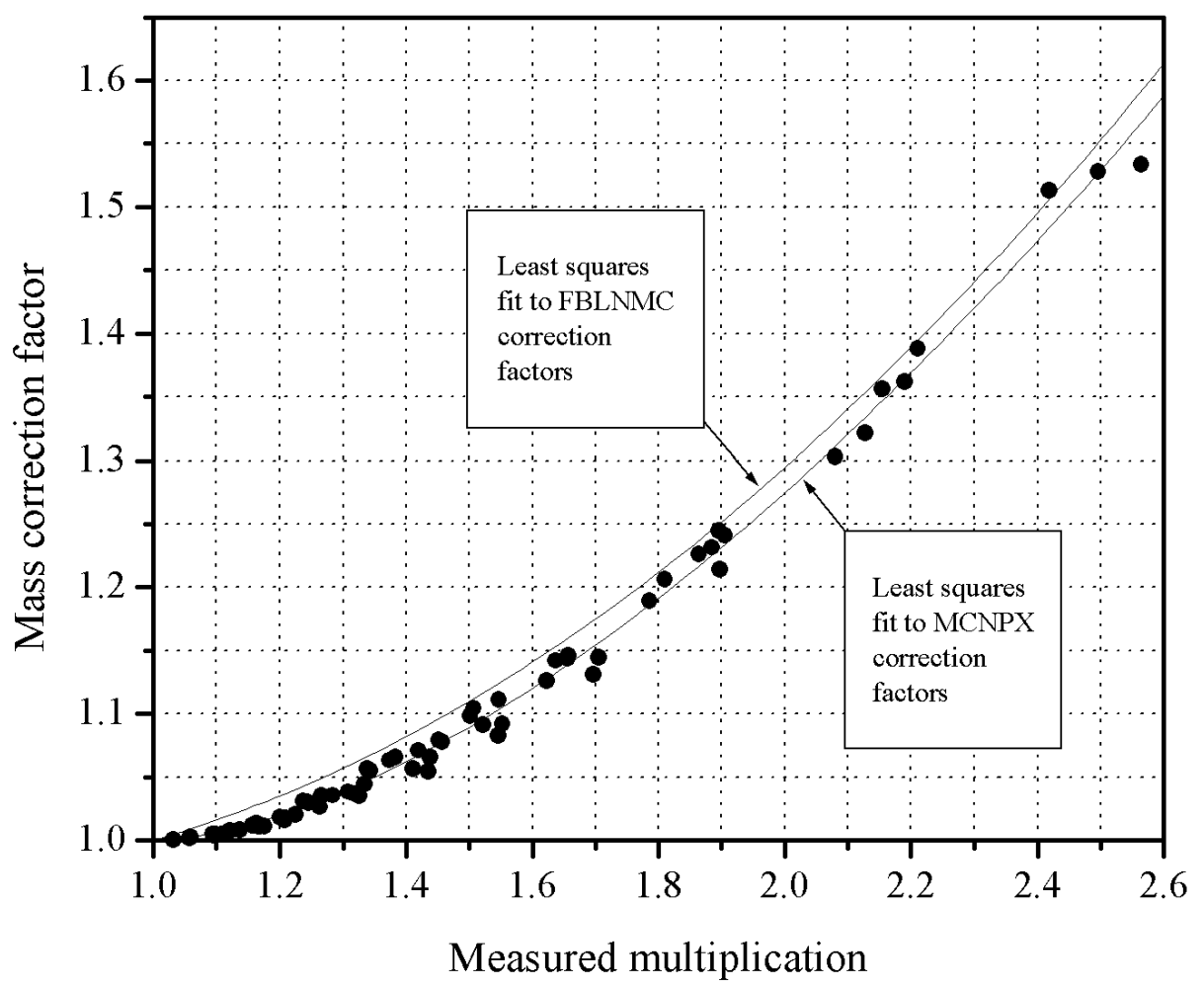

Fig. 18. Effective ${ }^{240} \mathrm{Pu}$ mass correction factor vs. measured multiplication for two sets of plutonium samples-one real and one computational. The computational set consists of the 66 cylinders used to determine the doubles and triples weighting factors, and the real set consists of 232 plutonium metal samples measured with the FBLNMC. The two curves are unweighted least squares fits to the two data sets. Only the MCNPX data points are plotted.

\section{Alpha dependence of the mass correction factors}

A comparison of the alpha dependence of the mass correction factors determined from the MCNPX and experimental data is shown in Fig. 19, where the mass correction factors are plotted vs. the measured alpha value. The MCNPX results are for a $2-\mathrm{kg}$ plutonium metal cylinder with diameter $=$ height and true alpha values of $0,0.2,0.4,0.6$, and 0.8 . The experimental results are from an evaluation of the alpha dependence for 10 wellcharacterized plutonium metal samples with masses from approximately 1.5- to 2-kg and measured alpha values from 0.13 to 1.28 [7]. The shapes of the measured samples are not known in detail, but are thought to be cast metal buttons with rounded bottoms; their measured multiplication values are similar to MCNPX multiplication values for the same masses in cylindrical shape with diameter $=$ height. The evaluated mass correction factor is

$$
f_{m}=f_{M} f_{\alpha},
$$


where the multiplication factor is

$$
f_{M}=a_{M}+b_{M}(M-1)+c_{M}(M-1)^{2},
$$

where

$$
\begin{aligned}
& a_{M}=1, \\
& b_{M}=0.06523,
\end{aligned}
$$

and

$$
c_{M}=0.14922
$$

and where the alpha factor is

$$
f_{\alpha}=1+a_{\alpha} \alpha+b_{\alpha} \alpha^{2}
$$

where

$$
a_{\alpha}=0.082078
$$

and

$$
b_{\alpha}=0.10166
$$

Although there is rough agreement between the experimental and Monte Carlo results, the experimental results show a much stronger dependence on alpha than the Monte Carlo results. However, so far the experimental dependence is based on only 10 samples, which have unknown impurities. 


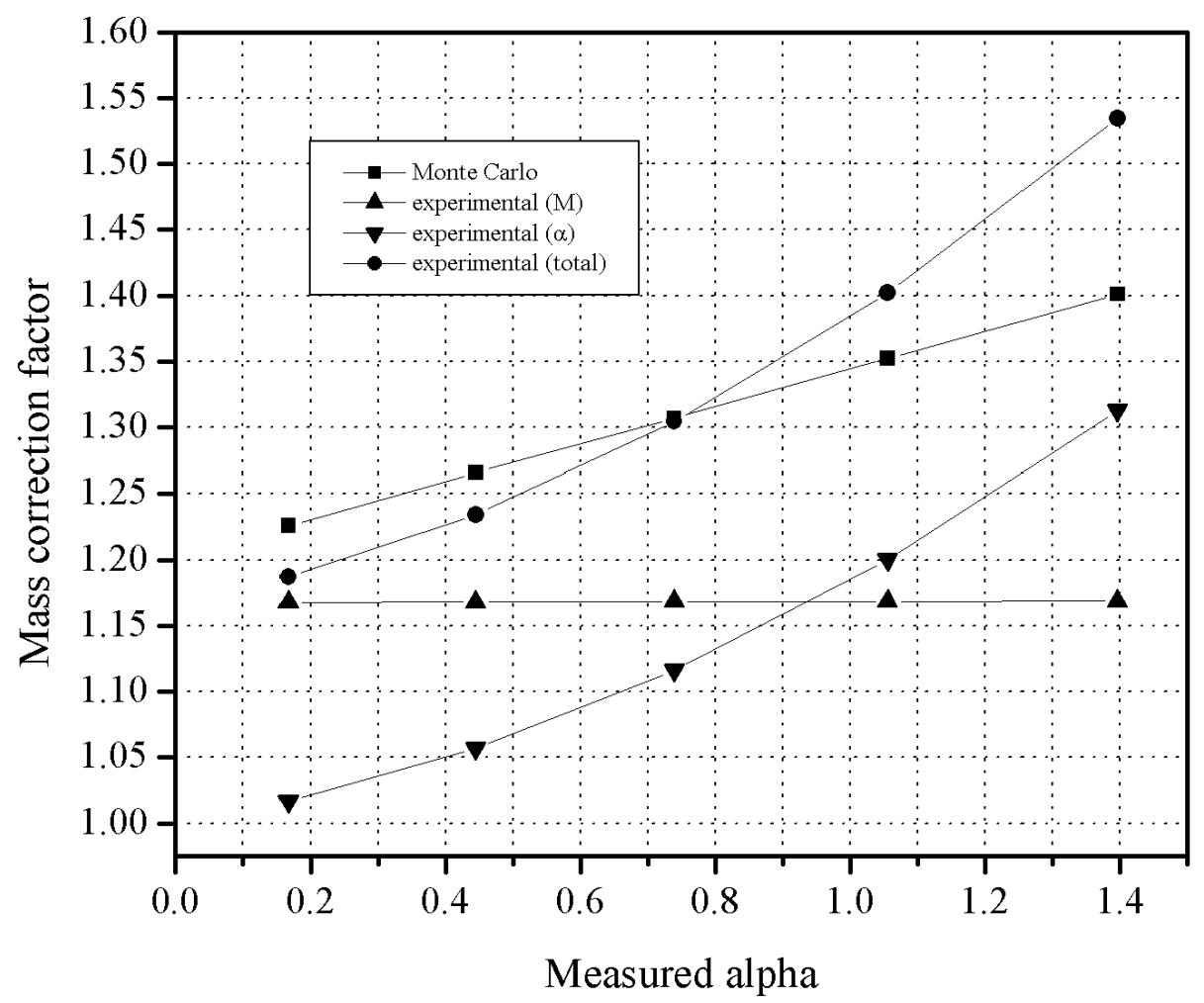

Fig. 19. Effective ${ }^{240} P u$ mass correction factor vs. measured alpha value from MCNPX and experimental data. The total experimental correction factor is the product of the multiplication and alpha correction factors. The MCNPX data were obtained for a 2-kg plutonium cylinder with diameter $=$ height, which has a similar multiplication to the experimental samples.

\section{Assay of Rocky Flats plutonium metal samples}

Data for 32 Rocky Flats plutonium metal samples measured in the FBLNMC were analyzed with the standard point model and the weighted point model. The samples had plutonium masses of approximately $2 \mathrm{~kg}$, multiplication values of approximately 1.4 , and assay alpha values mostly close to 0 . Fig. 20 shows the ratio of the assay to reference effective ${ }^{240} \mathrm{Pu}$ mass for the standard point model vs. the reference effective ${ }^{240} \mathrm{Pu}$. The average ratio is $0.934 \pm 0.005$. The errors of the individual points are not known, but are most likely dominated by errors in the isotopic composition, which contributes a standard deviation of 1.6\%. The same plot for the weighted point model is shown in Fig. 21, where the average ratio is $0.992 \pm 0.005$. The weighted point model, therefore, reduces a $-6.6 \pm$ $0.5 \%$ bias to a $-0.8 \pm 0.5 \%$ bias in this example. 


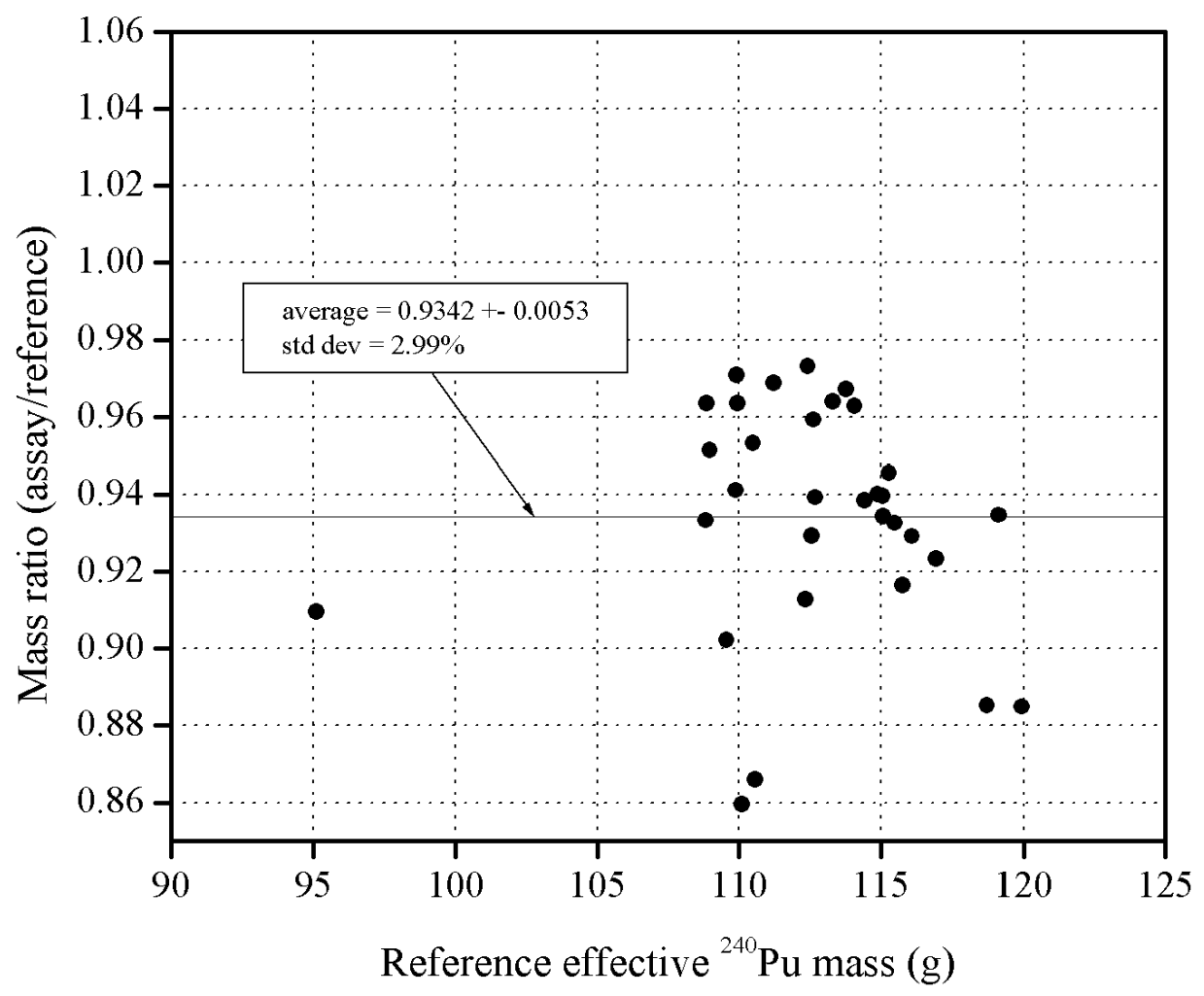

Fig. 20. Ratio of assay to reference effective ${ }^{240}$ Pu mass vs. reference effective ${ }^{240}$ Pu mass for 32 Rocky Flats samples measured in the FBLNMC and analyzed with the standard point model. The errors of the individual points are not known, but are likely dominated by measurements of the isotopic composition, which contributes a standard deviation of $1.6 \%$. 


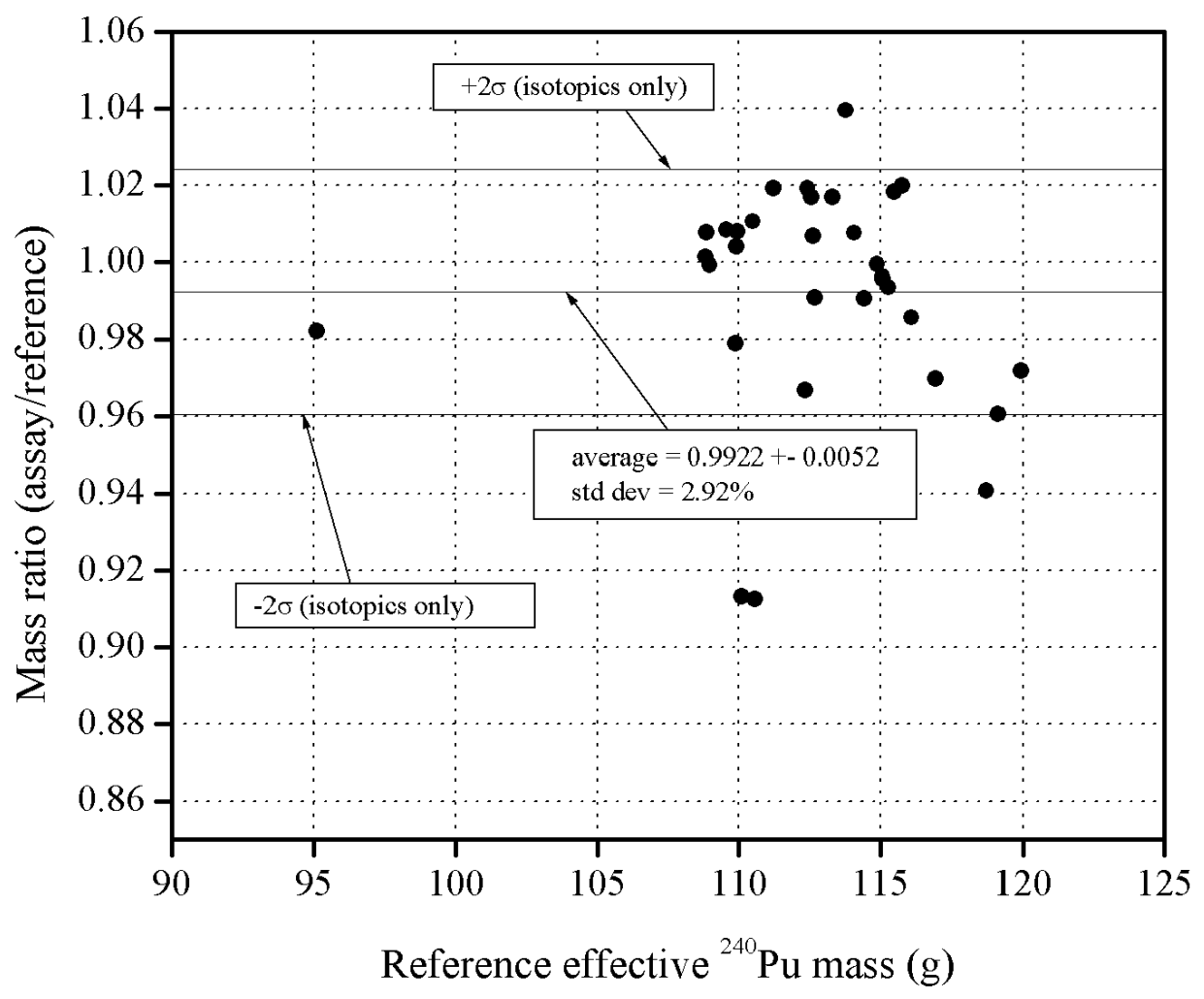

Fig. 21. Ratio of assay to reference effective ${ }^{240}$ Pu mass vs. reference effective ${ }^{240}$ Pu mass for 32 Rocky Flats samples measured in the FBLNMC and analyzed with the weighted point model. The errors of the individual points are not known, but are likely dominated by measurements of the isotopic composition, which contributes a standard deviation of $1.6 \%$. Only the corresponding $\pm 2 \sigma$ error band for isotopic composition is shown.

Fig. 22 shows the mass ratio for the weighted model plotted vs. the measured alpha value. Except for two samples, the alpha values are close to zero. It is interesting that the two samples with significant alpha values are biased by $-9 \%$ even with the weighted point model. The reason for this alpha-dependent bias is unknown, but the bias is consistent with effects covered in Section V. 


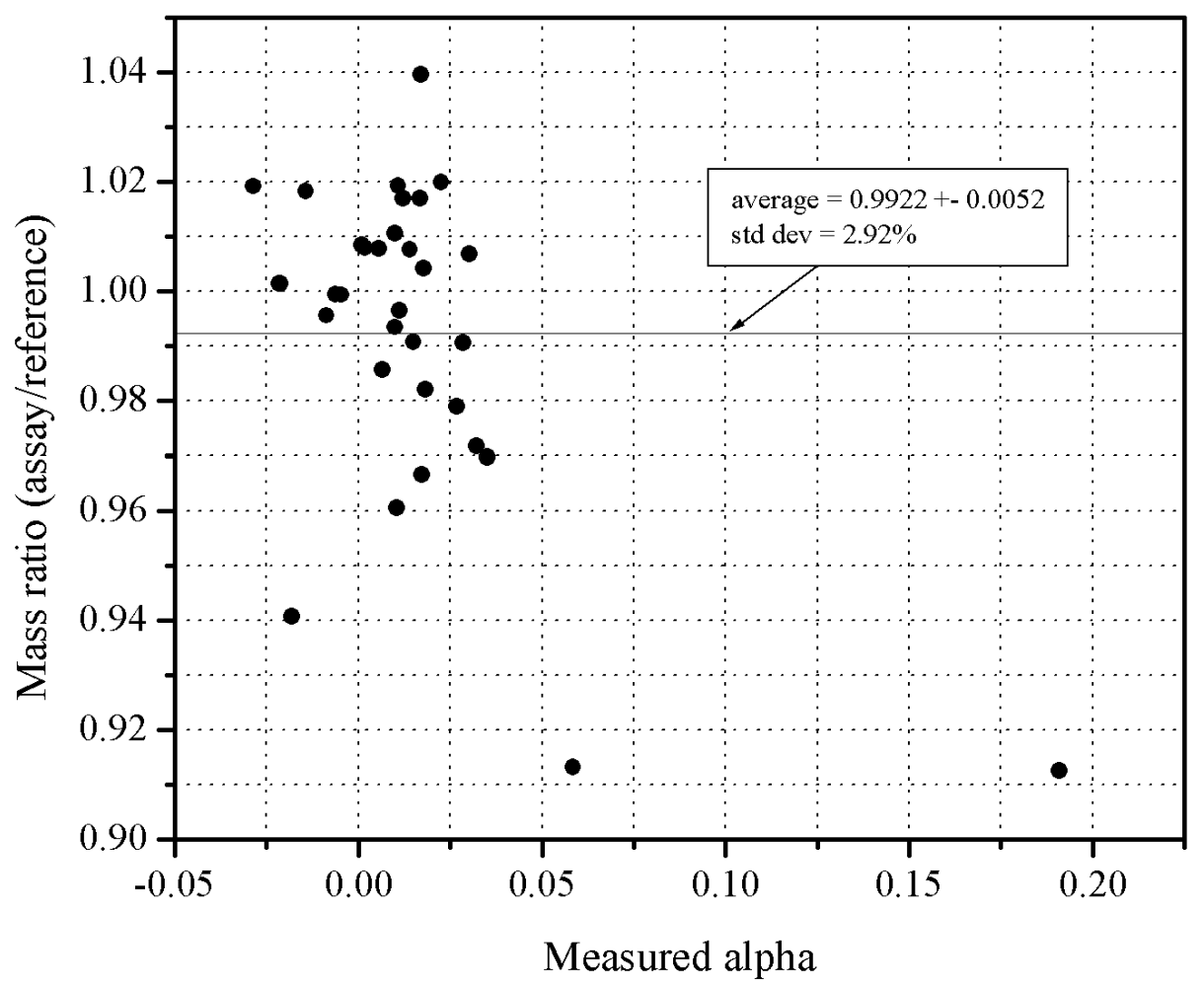

Fig. 22. Ratio of assay to reference effective ${ }^{240}$ Pu mass vs. measured alpha for 32 Rocky Flats samples measured in the FBLNMC and analyzed with the weighted point model.

\section{Assay of Savannah River plutonium metal samples}

Data for six well-characterized Savannah River plutonium metal samples [7] measured in the FBLNMC were analyzed with the standard and weighted point models. The samples had plutonium masses from approximately $1.1 \mathrm{~kg}$ to $2.1 \mathrm{~kg}$, multiplication values of approximately 1.7 , and alpha values from approximately 0 to 0.6 . The assay results are shown in Fig. 23, where the ratio of the assay to reference mass is plotted vs. the reference effective ${ }^{240} \mathrm{Pu}$ mass for both models. The plotted errors are standard deviations from the uncertainty in the isotopic compositions. The average mass ratio for the standard model is $0.840 \pm 0.026$ and for the weighted model is $1.020 \pm 0.022$. The weighted point model reduces a $-16.0 \pm 2.6 \%$ bias to a $2.0 \pm 2.2 \%$ bias in this example. However, when the mass ratios are plotted vs. the measured alpha values for the weighted point model in Fig. 24, an alpha-dependent bias is apparent. The reason for this alpha-dependent bias is unknown, but the bias is consistent with effects covered in section $\mathrm{V}$. 


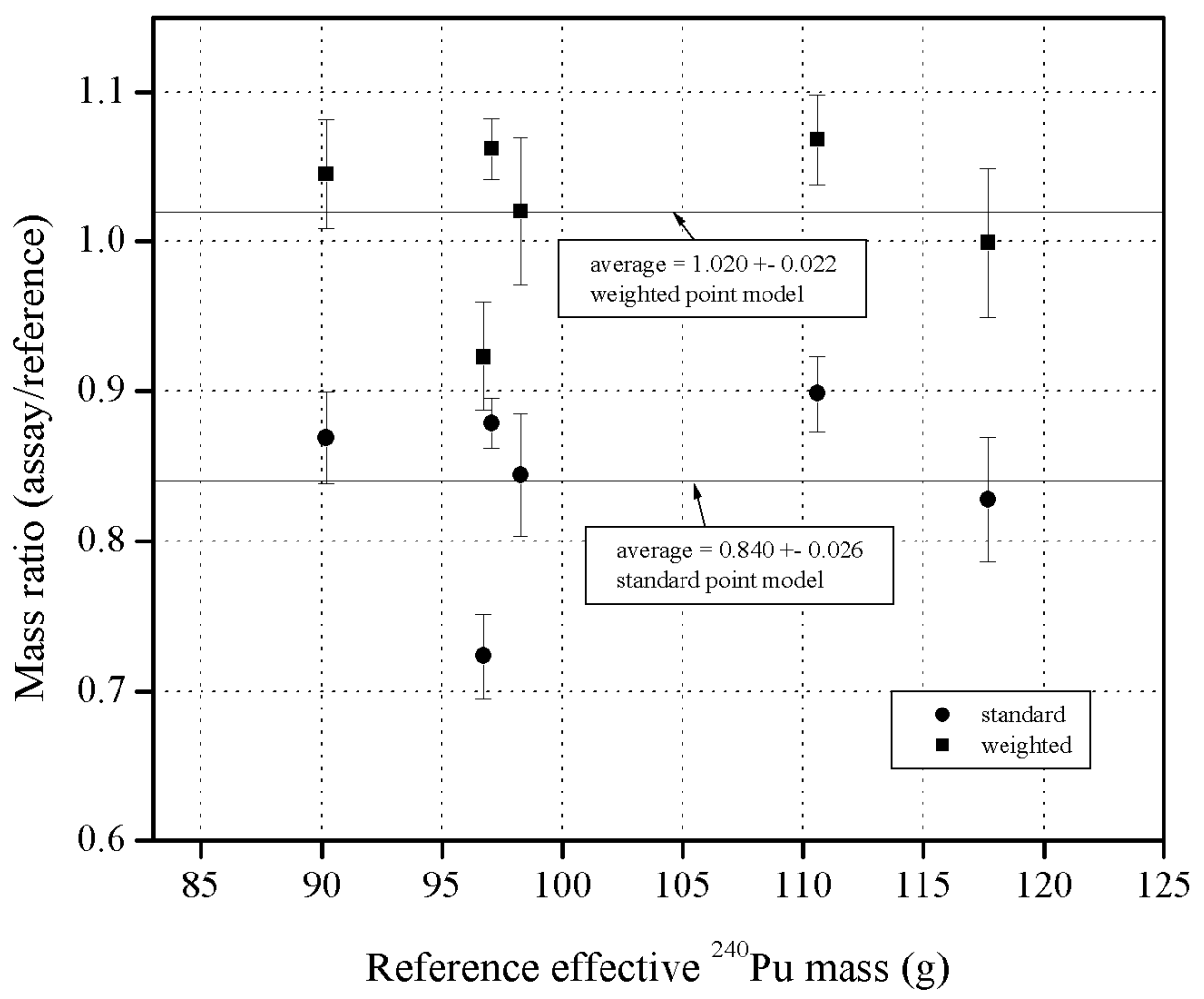

Fig. 23. Ratio of assay to reference effective ${ }^{240} P u$ mass vs. reference effective ${ }^{240} P u$ mass for six wellcharacterized Savannah River plutonium metal samples measured in the FBLNMC and analyzed with the standard and weighted point models. 


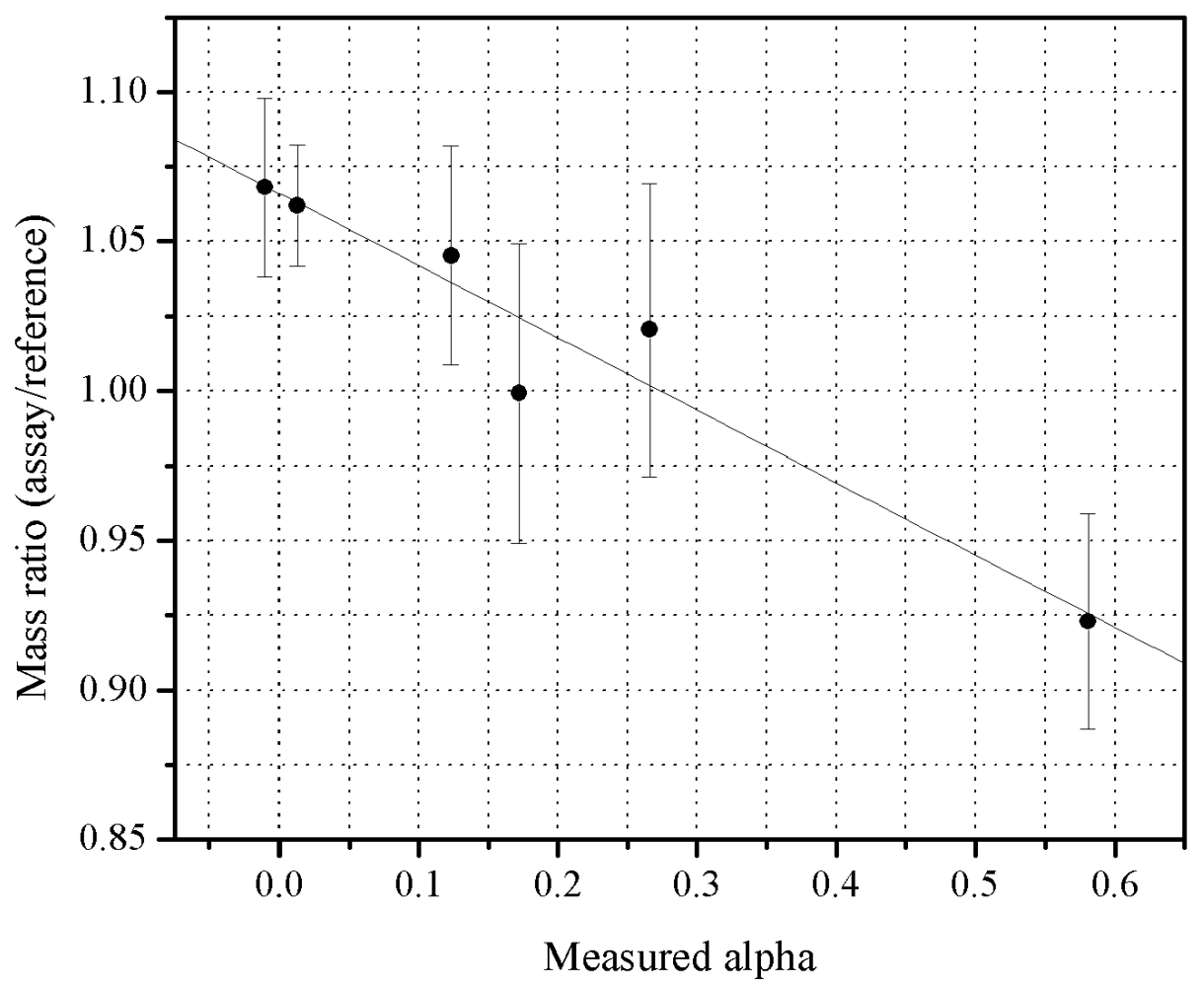

Fig. 24. Ratio of assay to reference effective ${ }^{240}$ Pu mass vs. measured alpha for six well-characterized Savannah River plutonium metal samples measured in the FBLNMC and analyzed with the weighted point model.

\section{FOUR-PARAMETER POINT MODEL MULTIPLICITY ANALYSIS}

The standard point model multiplicity equations that include the quadruple coincidences (quads) are potentially valuable because they would allow for the assay of samples that have four unknown quantities. The point model equations, including the quads, are [9]

$$
\begin{aligned}
& S=m F_{0} \varepsilon v_{s 1} M(1+\alpha), \\
& D=\frac{1}{2} m F_{0} \varepsilon^{2} f_{d} v_{s 2} M^{2}\left[1+\left(\frac{M-1}{v_{i 1}-1}\right) \frac{v_{s 1} v_{i 2}}{v_{s 2}}(1+\alpha)\right], \\
& T=\frac{1}{6} m F_{0} \varepsilon^{3} f_{t} v_{s 3} M^{3}\left\{1+\left(\frac{M-1}{v_{i 1}-1}\right) \frac{3 v_{s 2} v_{i 2}+v_{s 1} v_{i 3}(1+\alpha)}{v_{s 3}}+\right.
\end{aligned}
$$




$$
\begin{aligned}
\left.3\left(\frac{M-1}{v_{i 1}-1}\right)^{2} \frac{v_{s 1} v_{i 2}^{2}}{v_{s 3}}(1+\alpha)\right\} \\
Q=\frac{1}{24} m F_{0} \varepsilon^{4} f_{q} v_{s 4} M^{4}\left\{1+\left(\frac{M-1}{v_{i 1}-1}\right) \frac{6 v_{s 3} v_{i 2}+4 v_{s 2} v_{i 3}+v_{s 1} v_{i 4}(1+\alpha)}{v_{s 4}}+\right. \\
\left(\frac{M-1}{v_{i 1}-1}\right)^{2} \frac{15 v_{s 2} v_{i 2}^{2}+10 v_{s 1} v_{i 2} v_{i 3}(1+\alpha)}{v_{s 4}}+ \\
\left.15\left(\frac{M-1}{v_{i 1}-1}\right)^{3} \frac{v_{s 1} v_{i 2}^{3}(1+\alpha)}{v_{s 4}}\right\}
\end{aligned}
$$

where

$S, D, T, Q=$ singles, doubles, triples, and quads rates $(1 / \mathrm{s})$,

$m=$ effective ${ }^{240} \mathrm{Pu}$ mass $(\mathrm{g})$,

$M=$ neutron multiplication,

$\alpha=$ ratio of $(\alpha, n)$ to spontaneous fission neutrons,

$F_{0}={ }^{240} \mathrm{Pu}$ spontaneous fissions per gram ${ }^{240} \mathrm{Pu}$ per second

$\varepsilon=$ neutron detection efficiency,

$f_{d}, f_{t}, f_{q}=$ doubles, triples, and quads gate fractions,

$v_{s 1}, v_{s 2}, v_{s 3}, v_{s 4}=1$ st, 2nd, 3rd, and 4th factorial moments of the ${ }^{240} \mathrm{Pu}$ spontaneous fission neutron distribution,

$v_{i 1}, v_{i 2}, v_{i 3}, v_{i 4}=1$ st, 2nd, 3rd, and 4th factorial moments of the ${ }^{239} \mathrm{Pu}$ neutron-induced fission neutron distribution.

The weighted point model equations, including the quads, are

$$
\begin{aligned}
& S=m F_{0} \varepsilon v_{s 1} M(1+\alpha), \\
& D=\frac{1}{2} m F_{0} \varepsilon^{2} f_{d} v_{s 2} M^{2}\left(f_{D}+\alpha f_{D}^{\alpha}\right),
\end{aligned}
$$




$$
\begin{aligned}
& T=\frac{1}{6} m F_{0} \varepsilon^{3} f_{t} v_{s 3} M^{3}\left(f_{T}+\alpha f_{T}^{\alpha}\right), \\
& Q=\frac{1}{24} m F_{0} \varepsilon^{4} f_{q} v_{s 4} M^{4}\left(f_{Q}+\alpha f_{Q}^{\alpha}\right),
\end{aligned}
$$

where

$$
\begin{aligned}
& f_{D}=w_{D}\left[1+c_{1}(M-1)\right], \\
& f_{D}^{\alpha}=w_{D}^{\alpha} c_{1}(M-1), \\
& f_{T}=w_{T}\left[1+c_{2}(M-1)+c_{3}(M-1)^{2}\right], \\
& f_{T}^{\alpha}=w_{T}^{\alpha}\left[c_{4}(M-1)+c_{3}(M-1)^{2}\right], \\
& f_{Q}=w_{Q}\left[1+c_{5}(M-1)+c_{6}(M-1)^{2}+c_{7}(M-1)^{3}\right], \\
& f_{Q}^{\alpha}=w_{Q}^{\alpha}\left[c_{8}(M-1)+c_{9}(M-1)^{2}+c_{7}(M-1)^{3}\right],
\end{aligned}
$$

where

$$
\begin{aligned}
& c_{1}=\frac{v_{s 1} v_{i 2}}{v_{s 2}\left(v_{i 1}-1\right)}, \\
& c_{2}=\frac{3 v_{s 2} v_{i 2}+v_{s 1} v_{i 3}}{v_{s 3}\left(v_{i 1}-1\right)}, \\
& c_{3}=\frac{3 v_{s 1} v_{i 2}^{2}}{v_{s 3}\left(v_{i 1}-1\right)^{2}}, \\
& c_{4}=\frac{v_{s 1} v_{i 3}}{v_{s 3}\left(v_{i 1}-1\right)}, \\
& c_{5}=\frac{6 v_{s 3} v_{i 2}+4 v_{s 2} v_{i 3}+v_{s 1} v_{i 4}}{v_{s 4}\left(v_{i 1}-1\right)}, \\
& c_{6}=\frac{15 v_{s 2} v_{i 2}^{2}+10 v_{s 1} v_{i 2} v_{i 3}}{v_{s 4}\left(v_{i 1}-1\right)^{2}}, \\
& c_{7}=\frac{15 v_{s 1} v_{i 2}^{3}}{v_{s 4}\left(v_{i 1}-1\right)^{3}},
\end{aligned}
$$




$$
\begin{aligned}
& c_{8}=\frac{v_{s 1} v_{i 4}}{v_{s 4}\left(v_{i 1}-1\right)}, \\
& c_{9}=\frac{10 v_{s 1} v_{i 2} v_{i 3}}{v_{s 4}\left(v_{i 1}-1\right)^{2}},
\end{aligned}
$$

where $w_{D}, w_{D}^{\alpha}, w_{T}, w_{T}^{\alpha}, w_{Q}$, and $w_{Q}^{\alpha}$ are the variable-multiplication weighting factors. The other symbols have the same meanings as described above.

The spontaneous fission and $(\alpha, n)$ contributions to the doubles, triples, and quads rates are all different functions of the multiplication and therefore have different weighting factors. The singles rate is proportional to the multiplication and so does not need weighting factors. When the six weighting factors are set to unity, the weighted point model equations become the standard point model equations.

Because a plutonium metal sample with a nonuniform $(\alpha, n)$ source distribution produces a bias when assayed with the three-parameter weighted point model equations, such a sample was used as a test case for the use of the weighted four-parameter equations. To simplify the problem, it was assumed that the $(\alpha, n)$ source was distributed uniformly over some fraction $f$ (the fill fraction) of a cylindrical plutonium metal sample such that the $(\alpha, n)$ source extended the full diameter of the cylinder but only the fraction $f$ of the full length, starting at one end. The goal was to solve the four multiplicity equations for $m$, $M, \alpha$, and $f$ and see if the determination of $f$ improved the assay mass. The multiplication $M$ now refers to the multiplication of the uniform spontaneous fission source only; the multiplication of the nonuniform $(\alpha, n)$ source will in general be different.

The four-parameter weighted point model multiplicity equations were modified for the nonuniform $(\alpha, n)$ source as follows:

$$
\begin{aligned}
& S=m F_{0} \varepsilon v_{s 1} M\left(1+u_{S}^{\alpha} \alpha\right), \\
& D=\frac{1}{2} m F_{0} \varepsilon^{2} f_{d} v_{s 2} M^{2}\left(f_{D}+\alpha f_{D}^{\alpha}\right), \\
& T=\frac{1}{6} m F_{0} \varepsilon^{3} f_{t} v_{s 3} M^{3}\left(f_{T}+\alpha f_{T}^{\alpha}\right), \\
& Q=\frac{1}{24} m F_{0} \varepsilon^{4} f_{q} v_{s 4} M^{4}\left(f_{Q}+\alpha f_{Q}^{\alpha}\right),
\end{aligned}
$$

where

$$
f_{D}=w_{D}\left[1+c_{1}(M-1)\right]
$$




$$
\begin{aligned}
& f_{D}^{\alpha}=u_{D}^{\alpha} w_{D}^{\alpha} c_{1}(M-1), \\
& f_{T}=w_{T}\left[1+c_{2}(M-1)+c_{3}(M-1)^{2}\right], \\
& f_{T}^{\alpha}=u_{T}^{\alpha} w_{T}^{\alpha}\left[c_{4}(M-1)+c_{3}(M-1)^{2}\right], \\
& f_{Q}=w_{Q}\left[1+c_{5}(M-1)+c_{6}(M-1)^{2}+c_{7}(M-1)^{3}\right], \\
& f_{Q}^{\alpha}=u_{Q}^{\alpha} w_{Q}^{\alpha}\left[c_{8}(M-1)+c_{9}(M-1)^{2}+c_{7}(M-1)^{3}\right],
\end{aligned}
$$

and where $u_{S}^{\alpha}, u_{D}^{\alpha}, u_{T}^{\alpha}$, and $u_{Q}^{\alpha}$ are weighting factors for the nonuniform $(\alpha, \mathrm{n})$ distribution. The other symbols have the same meanings as described above.

The variable-multiplication weighting factors for the quads $\left[w_{Q}\right.$ for a spontaneous fission source and $w_{Q}^{\alpha}$ for an $(\alpha, n)$ source] were obtained from the same 66 MCNPX runs that were used to get the doubles and triples weighting factors. The results for $w_{Q}$ and $w_{Q}^{\alpha}$ are shown in Figs. 25 and 26, respectively, together with unweighted least-squares fits. Numerically,

$$
w_{Q}=1+0.5198(M-1)+0.0746(M-1)^{2}
$$

and

$$
w_{Q}^{\alpha}=1+0.6682(M-1) .
$$




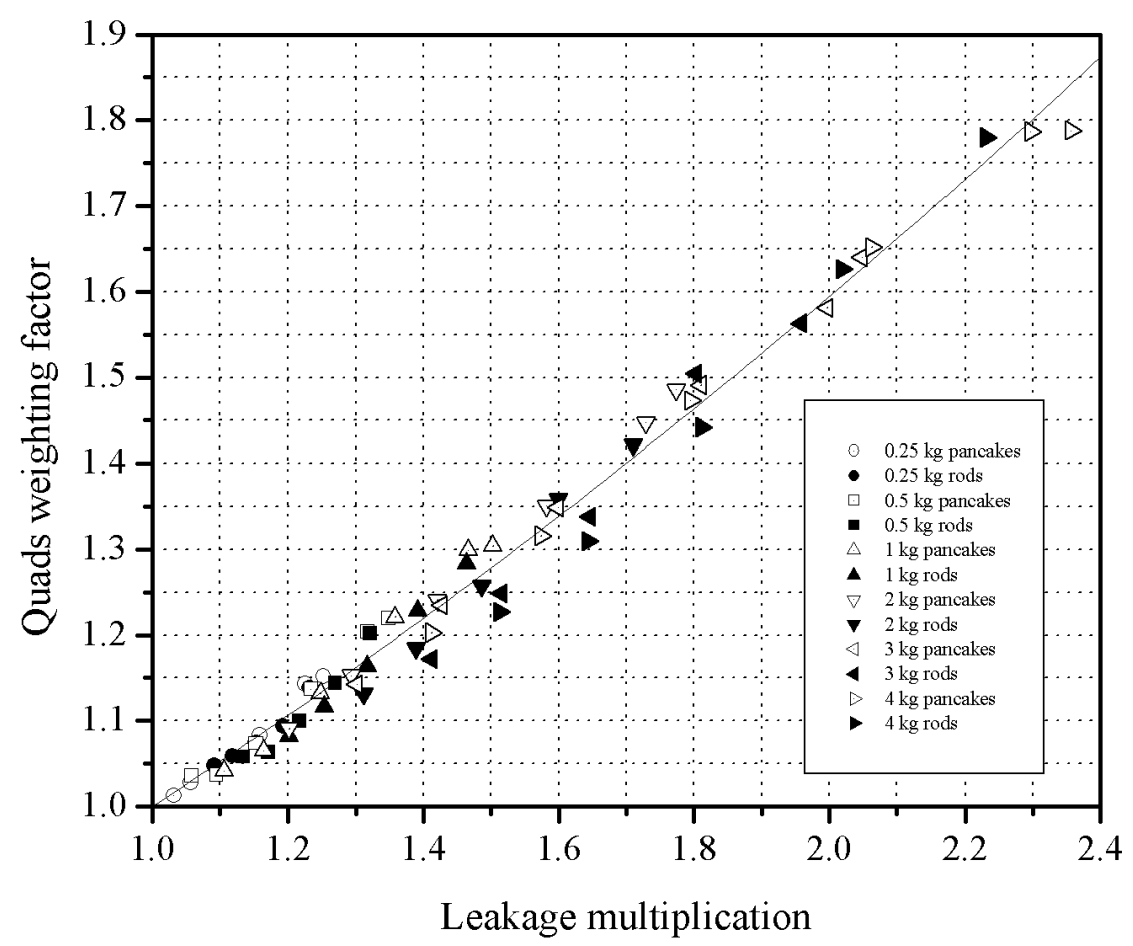

Fig. 25. Quads weighting factor vs. leakage multiplication for plutonium metal cylinders with a ${ }^{240} P u$ spontaneous fission source and masses from 0.25 to $4 \mathrm{~kg}$. Eleven height/diameter ratios are plotted for each mass. The solid line is an unweighted least-squares fit to the 66 data points. 


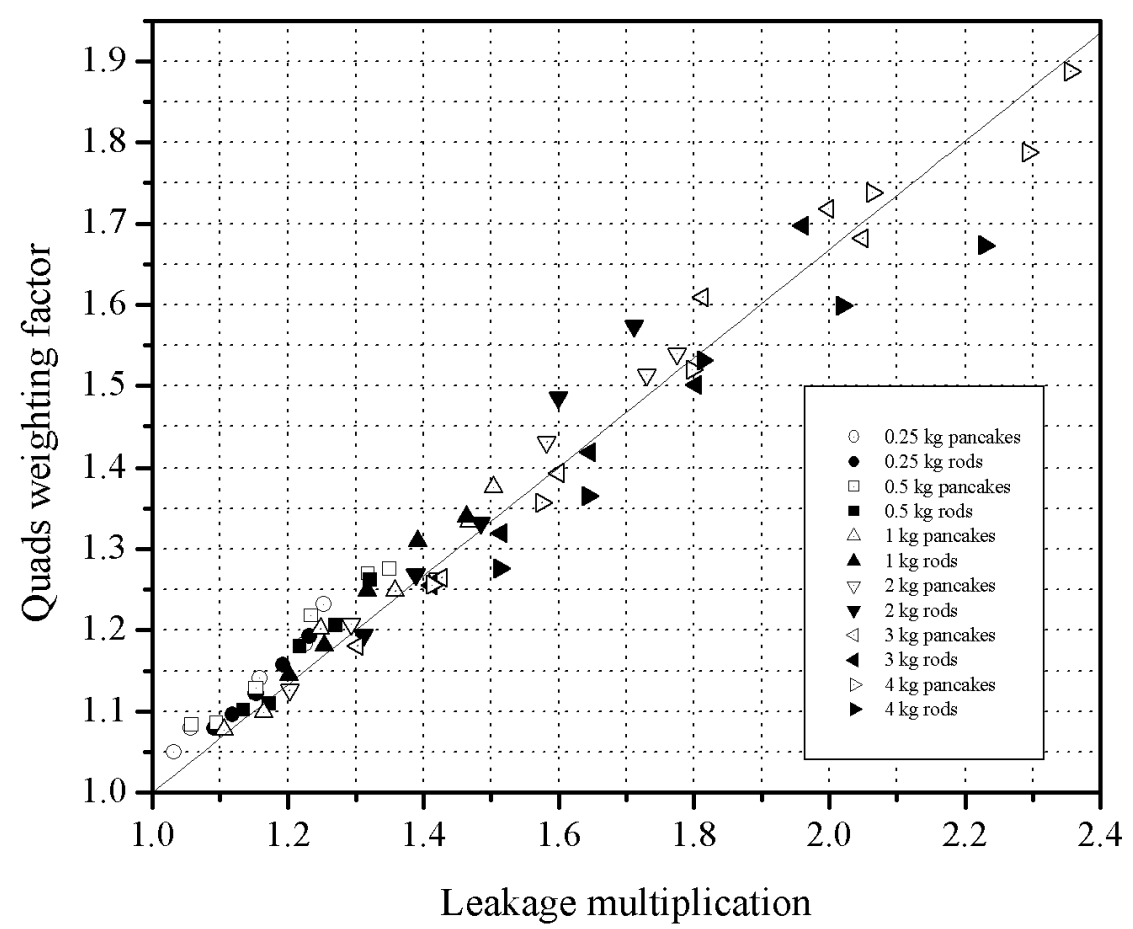

Fig. 26. Quads weighting factor vs. leakage multiplication for plutonium metal cylinders with masses from 0.25 to $4 \mathrm{~kg}$. The source is an $(\alpha, n)$ source with ${ }^{240} P u$ spontaneous fission neutron energy spectrum. Eleven height/diameter ratios are plotted for each mass. The solid line is an unweighted least squares fit to the 66 data points.

Additional MCNPX runs were done to obtain the nonuniform $(\alpha, n)$ weighting factors $u_{S}^{\alpha}, u_{D}^{\alpha}, u_{T}^{\alpha}$, and $u_{Q}^{\alpha}$. These weighting factors are functions of $M$ and $f$, but were determined as a function of $f$ only for a test case, which was a $2-\mathrm{kg}$ plutonium metal cylinder with diameter $=$ height and a variable fill fraction. The $(\alpha, n)$ source has a ${ }^{240} \mathrm{Pu}$ spontaneous fission neutron energy spectrum. Fig. 27 shows the four weighting factors vs. the fill fraction, including the MCNPX data points at $f=0.1,0.2$, and 0.3 and the unweighted least-squares fits forced to unity at $f=1$. Numerically,

$$
\begin{aligned}
& u_{S}^{\alpha}=0.8296+0.5380 f-0.3676 f^{2}, \\
& u_{D}^{\alpha}=0.5477+1.384 f-0.9315 f^{2}, \\
& u_{T}^{\alpha}=0.5076+1.464 f-0.9715 f^{2}, \\
& u_{Q}^{\alpha}=0.4975+1.448 f-0.9455 f^{2} .
\end{aligned}
$$




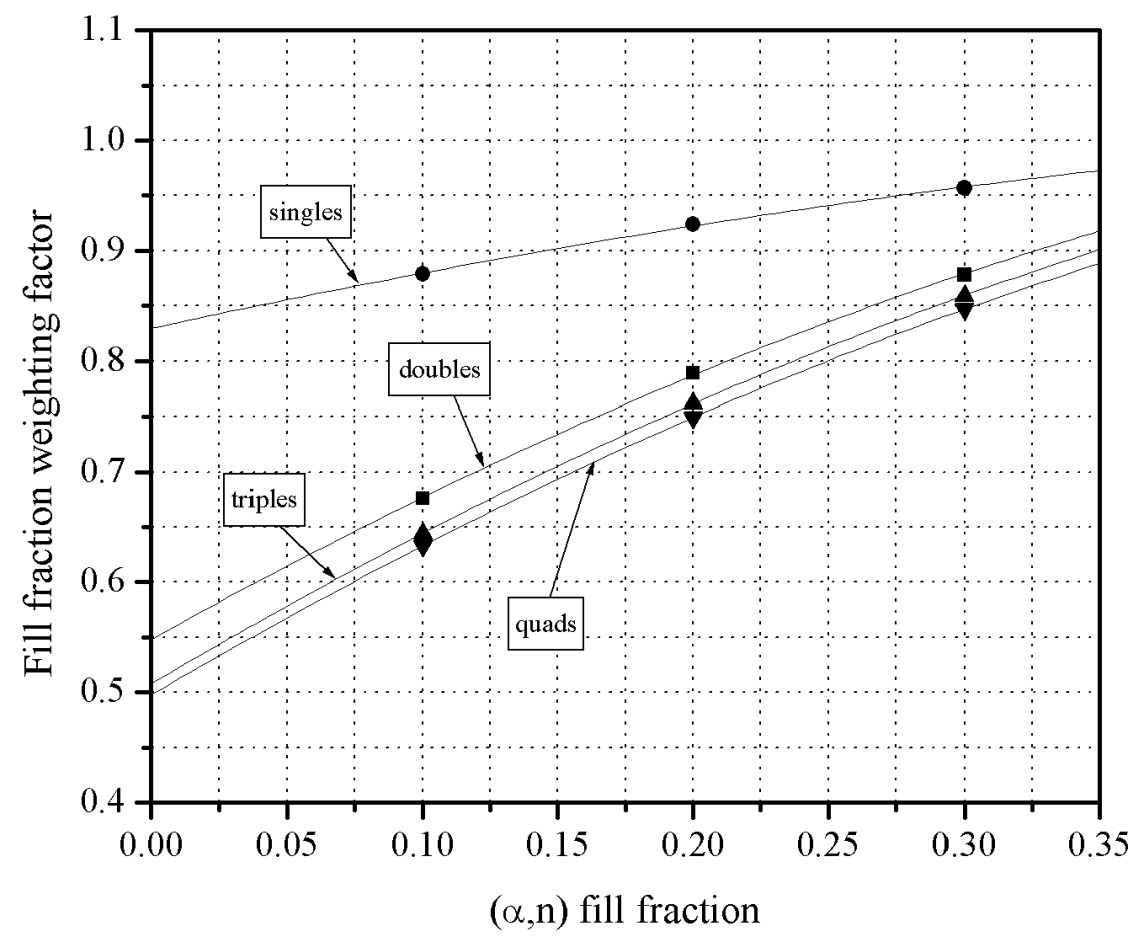

Fig. 27. Fill fraction weighting factors for $S, D, T$, and $Q v s$. the $(\alpha, n)$ fill fraction (f) for a 2-kg plutonium metal cylinder with diameter $=$ height. The $(\alpha, n)$ source has $a{ }^{240} P u$ spontaneous fission neutron energy spectrum and fills the fraction fof the cylinder uniformly across the diameter and along the length for a fraction fof the full length starting at one end. The plotted points are from MCNPX calculations, and the solid curves are unweighted least squares fits to the MCNPX points. The curves are forced to unity at

$$
f=1 \text {. }
$$

Once the weighting factors were determined, the quad equations could be solved for $m$, $M, \alpha$, and $f$ given the detector parameters and the rates $S, D, T$, and $Q$. The detector efficiency and gate fractions were all set to 1 . A test case was run for a $2-\mathrm{kg}$ plutonium metal cylinder with diameter $=$ height $(M=1.774)$, with $\alpha=1$ and with $f=0.2$. The rates are all proportional to $m$, so $m$ was arbitrarily set to 1 . Inserting these values into the quad equations above gives the rates shown in Table $\mathrm{V}$ as the weighted point model rates. A direct MCNPX run with the same sample parameters gave the rates shown in Table V as the MCNPX rates. The ratios of these two sets of rates are also shown in Table V. The $S$, $D, T$, and $Q$ rates all agree to within $1 \%$. 
Table V. The $S, D, T$, and $Q$ rates for a $2-\mathrm{kg}$ Pu cylinder with $m=1, M=1.774, \alpha=1$, and $f=0.2$ from the weighted point model equations and directly from MCNPX calculations.

\begin{tabular}{|l|c|c|c|c|}
\hline & $S$ & $D$ & $T$ & $Q$ \\
\hline Weighted point model rates (1/s) & 3477 & 12282 & 90273 & 854867 \\
\hline MCNPX rates (1/s) & 3479 & 12326 & 90980 & 859617 \\
\hline Ratio (MCNPX/model) & 1.001 & 1.004 & 1.008 & 1.006 \\
\hline
\end{tabular}

The quad equations were then solved for $m, M, \alpha$, and $f$ using the two sets of rates from Table V. The results are shown in Table VI. The assay results from the weighted point model rates agree with the sample specifications, as expected. However, the MCNPX rates produce $\mathrm{a}-13.3 \%$ mass error, showing that the assay results are very sensitive to the input rates.

Table VI. Assay results from the weighted point model quad equations using the $S, D, T$, and $Q$ rates from the same equations and from direct MCNPX calculations.

\begin{tabular}{|l|c|c|c|c|}
\hline & $m$ & $M$ & $\alpha$ & $f$ \\
\hline Assay from weighted point model rates (1/s) & 1.000 & 1.774 & 1.000 & 0.200 \\
\hline Assay from MCNPX rates (1/s) & 0.867 & 1.772 & 1.246 & 0.373 \\
\hline
\end{tabular}

This sensitivity was studied more carefully by increasing each of the weighted point model count rates separately by $0.5 \%$ and obtaining the assay values in each case. The results are shown in Table VII. In particular, a $0.5 \%$ increase in the triples rate results in a $-21.6 \%$ error in assay mass and a $147 \%$ error in the fill fraction.

Table VII. Assay results obtained by increasing the weighted point model reference rates separately by $0.5 \%$.

\begin{tabular}{|l|c|c|c|c|}
\hline & $m$ & $M$ & $\alpha$ & $f$ \\
\hline Reference values & 1.000 & 1.774 & 1.000 & 0.200 \\
\hline Assay results $(S:+0.5 \%)$ & 0.989 & 1.774 & 1.034 & 0.199 \\
\hline Assay results $(D:+0.5 \%)$ & 1.131 & 1.774 & 0.802 & 0.083 \\
\hline Assay results $(T:+0.5 \%)$ & 0.784 & 1.770 & 1.448 & 0.493 \\
\hline Assay results $(Q:+0.5 \%)$ & 1.098 & 1.777 & 0.856 & 0.086 \\
\hline
\end{tabular}

Fig. 28 shows the quantity $D Q / T^{2}$ vs. the leakage multiplication for three sets of plutonium metal samples: (1) $2-\mathrm{kg}$ cylinders with various height/diameter ratios and $\alpha=$ 0 , (2) cylinders with diameter $=$ height, masses ranging from 0.25 to $4 \mathrm{~kg}$, and $\alpha=0$, and (3) $2-\mathrm{kg}$ cylinders with diameter $=$ height, various $(\alpha, \mathrm{n})$ fill fractions, and $\alpha=1$. The $(\alpha, n)$ source has a ${ }^{240} \mathrm{Pu}$ spontaneous fission neutron energy spectrum. The unweighted 
average of $D Q / T^{2}$ over all these samples is 1.283 ; the standard deviation is $1.0 \%$. Thus, for this group of samples, the quantity $1.283 T^{2} / D$ determines $Q$ to about $1 \%$. This implies that it will be difficult to solve for a fourth unknown quantity from the measured value of the quads for these samples. However, it is easy to obtain fractional percent precision in the measurement of $S, D, T$, and $Q$ for highly multiplying samples, so the quads offer a possibility of extracting additional information from the measurements.

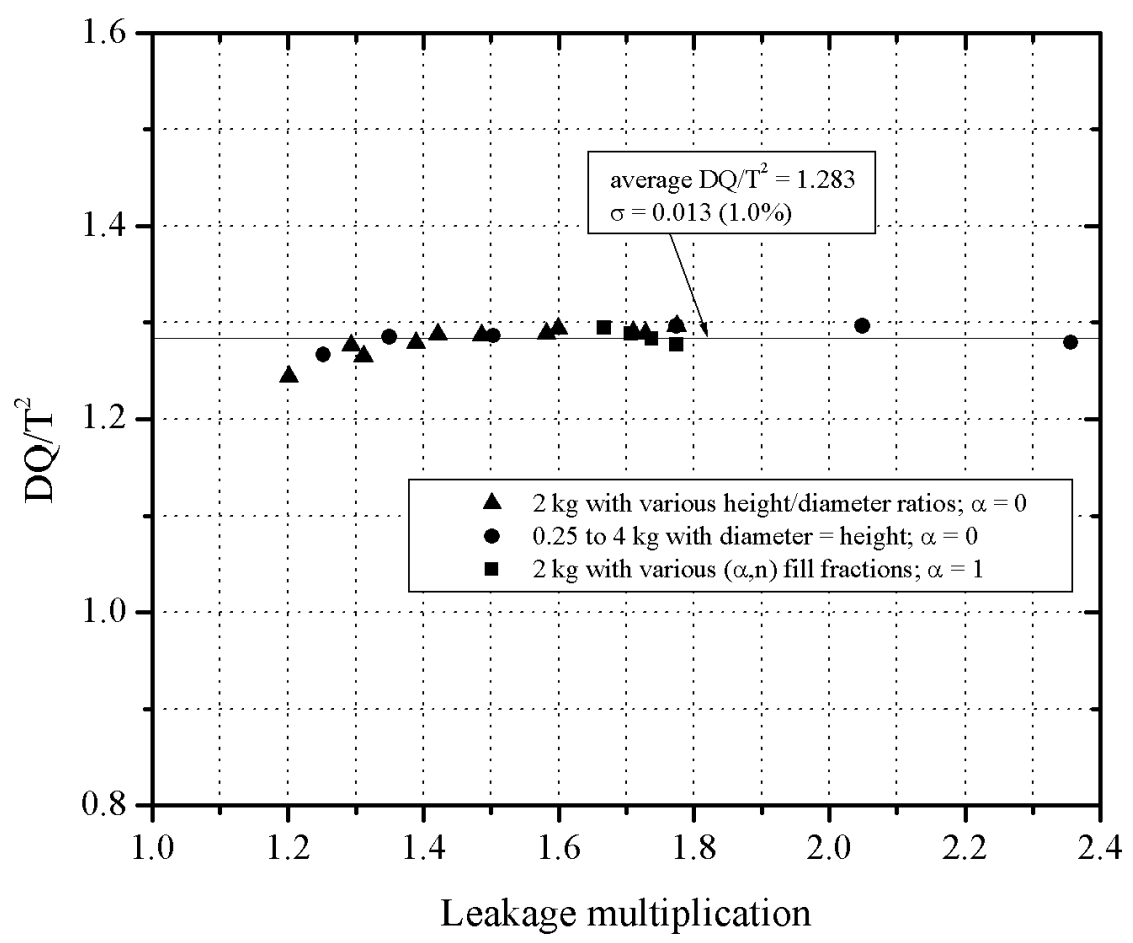

Fig. 28. The ratio $D Q / T^{2}$ vs. leakage multiplication for three sets of plutonium metal samples. The $(\alpha, n)$ source has $a^{240} \mathrm{Pu}$ spontaneous fission neutron energy spectrum.

A test case with $m=120 \mathrm{~g}\left(2-\mathrm{kg}\right.$ Pu with $\left.6 \%{ }^{240} \mathrm{Pu}\right), M=1.774, \alpha=1$, and $f=0.2$ was studied by performing custom Monte Carlo simulations of the test sample in a typical thermal neutron multiplicity counter with $\varepsilon=0.5$, predelay $=3 \mathrm{~s}$, gate length $=32 \mathrm{~s}$, and dieaway time $=50 \mathrm{~s}$. The neutron source distribution was obtained from MCNPX, and the detected neutron pulse stream was obtained from the custom code. A series of 160 1000 -s measurements was simulated to determine the measurement errors to a precision of about $6 \%$. The results are shown in Table VIII. 
Table VIII. Standard deviations of several quantities for simulated 1000-s measurements of the test sample in a typical thermal neutron multiplicity counter.

\begin{tabular}{|c|c|}
\hline Quantity & Standard deviation (\%) \\
\hline$S$ & 0.014 \\
\hline$D$ & 0.087 \\
\hline$T$ & 0.27 \\
\hline$Q$ & 0.68 \\
\hline$D Q / T^{2}$ & 0.33 \\
\hline$m$ & 6.6 \\
\hline
\end{tabular}

The assay mass is determined to a precision of $6.6 \%$ in $1000 \mathrm{~s}$ or $3.5 \%$ in an hour. Thus, if the weighting factors are well calibrated for the sample type being assayed, quad analysis might prove useful for improving assay accuracy. For example, if the weighted point model is used with the measured $S, D$, and $T$ rates, then for the test case with $m=$ $120 \mathrm{~g}, M=1.774, \alpha=1$, and $f=0.2$, the assay mass error is $-18 \%$ from Fig. 17 . If the measured $S, D, T$, and $Q$ rates are used with the quad equations, then a 1-h measurement will produce a mass standard deviation of about $3.5 \%$. Thus, if the weighting factors are accurate, the mass error can be reduced in this situation.

\section{CONCLUSIONS}

The negative biases observed in the assay of plutonium metal samples are primarily caused by variations in the neutron multiplication for neutrons originating at various locations in the sample. The bias depends on the mass and shape of the sample and depends on the amount and distribution of the $(\alpha, n)$ neutrons in the sample. When the standard point model is used, this variable-multiplication bias overestimates the multiplication and alpha values of the sample and underestimates the plutonium mass.

The MCNPX Monte Carlo code includes the capability to use spontaneous fission neutron sources and to tally the neutron multiplicity distributions of the detected neutrons. These features make possible for the first time the calculation of the variablemultiplication biases in the neutron multiplicity assay of plutonium samples. Thus the MCNPX code is of great importance for the development of nondestructive assay techniques for thermal neutron multiplicity counting.

MCNPX calculations have shown that the variable-multiplication bias is closely related to the average true neutron multiplication of the sample, but is insensitive to the mass and geometry of the sample for a specified multiplication. This behavior makes possible the use of multiplication correction factors for the doubles and triples rates from spontaneous fission and $(\alpha, n)$ sources that depend only on the neutron multiplication. A weighted point model for neutron multiplicity assay that includes these weighting factors provides an assay technique to account for the variable-multiplication effects in plutonium metal samples. 
The weighted point model potentially can provide assay accuracy of $\sim 2 \%$ ( $1 \sigma$ ) for cylindrical, homogeneous plutonium metal samples up to $4 \mathrm{~kg}$ with $\alpha<1$ without knowing the exact shape of the samples. Similar geometries will give good results also; e.g., plutonium spheres up to $2 \mathrm{~kg}$ will assay well using the weighting factors derived for cylinders. Better assay results can be obtained if there is some knowledge of the plutonium geometry because weighting factor curves can be calculated for any specified geometry.

Assay results are sensitive to the detector parameters used for the assay, so it is important to determine the parameters for the detector very well. Also, assay results depend on the $(\alpha, n)$ neutron energies, so the weighted point model may need extension to include energy-dependent $(\alpha, n)$ neutron sources.

Based on a very small data set, there can be an alpha-dependent bias in the weighted point model assay masses. Possible reasons for this include inaccurate detector parameters, $(\alpha, n)$ neutron energies different from fission neutron energies, and nonuniform $(\alpha, n)$ distributions. Ring ratios (the ratios of the singles count rates in the inner to outer detector rings) should be measured whenever possible to estimate the $(\alpha, n)$ neutron energies so that bias corrections can be made. Fortunately, most plutonium metal samples have low $\alpha$ values, so the alpha-dependent bias is not large.

It appears to be difficult to use the quads rates to obtain a fourth assay parameter for high-mass plutonium metal samples because the quads rate can be estimated very well from the doubles and triples rates. However, the measurement precision is very good, so improved assays are potentially possible with well-determined weighting factors.

A direct comparison of MCNPX and measured $S, D$, and $T$ rates is needed for several pure plutonium samples with well-known masses, shapes, and isotopic compositions to further validate MCNPX calculations for simulating neutron multiplicity measurements.

\section{REFERENCES}

1. N. Ensslin, et al., "Application Guide to Neutron Multiplicity Counting," Los Alamos National Laboratory report LA-13422-M (November 1998).

2. D. M. Cifarelli and W. Hage, "Models for a Three Parameter Analysis of Neutron Signal Correlation Measurements for Fissile Material Assay," Nuclear Instruments and Methods A251, 550 (1986).

3. M. S. Krick, D. G. Langner, and J. E. Stewart, "Energy-Dependent Bias in Plutonium Verification Measurements Using Thermal Neutron Multiplicity Counters," Los Alamos National Laboratory document LA-UR-97-3427 (1997). 
4. M. S. Krick, et al., "The IAEA Neutron Coincidence Counting (INCC) and the DEMING Least-Squares Fitting Programs," Los Alamos National Laboratory document LA-UR-98-2378 (1998).

5. J. S. Hendricks, et al., "MCNPX, Version 2.5.B," Los Alamos National Laboratory document LA-UR-02-7086 (November 2002).

6. D. G. Langner, et al., "Thermal Neutron Multiplicity Measurements Using the Pyrochemical Multiplicity Counter at Lawrence Livermore National Laboratory," Los Alamos National Laboratory document LA-UR-93-2610 (1993).

7. W. H. Geist, D. G. Langner, and N. Ensslin, "Analysis of FB-Line Neutron Multiplicity Counter Data," Los Alamos National Laboratory document LA-UR00-5792 (2000).

8. D. G. Langner, et al., "FB-Line Neutron Multiplicity Counter Operation Manual," Los Alamos National Laboratory report LA-13395-M (January 1998).

9. K. Boehnel, "The Effect of Multiplication on the Quantitative Determination of Spontaneously Fissioning Isotopes by Neutron Correlation Analysis," Nuclear Science and Engineering 90, 75-82 (1985). 
This report has been reproduced directly from the best available copy. It is available electronically on the Web (http://www.doe.gov/bridge).

Copies are available for sale to U.S. Department of Energy employees and contractors from:

Office of Scientific and Technical Information P.O. Box 62

Oak Ridge, TN 37831

(865) 576-8401

Copies are available for sale to the public from: National Technical Information Service

U.S. Department of Commerce

5285 Port Royal Road

Springfield, VA 22161

(800) 553-6847 


\section{Los Alamos}

NATIONAL LABORATORY

EST.1943 